\title{
Modeling the EUV spectra of optically thick boundary layers of dwarf novae in outburst
}

\author{
V. Suleimanov ${ }^{1,2}$, M. Hertfelder ${ }^{1}$, K. Werner ${ }^{1}$, and W. Kley ${ }^{1}$ \\ 1 Institut für Astronomie und Astrophysik, Kepler Center for Astro and Particle Physics, Universität Tübingen, Sand 1, \\ 72076 Tübingen, Germany \\ e-mail: suleimanov@astro.uni-tuebingen.de \\ 2 Kazan (Volga region) Federal University, Kremlevskaja str. 18, 420008 Kazan, Russia
}

Received 27 February 2014 / Accepted 14 August 2014

\begin{abstract}
Context. Disk accretion onto weakly magnetized white dwarfs (WDs) in cataclysmic variables (CVs) leads to the formation of a boundary layer (BL) between the accretion disk and the WD, where the accreted matter loses its excess kinetic energy and angular momentum. It is assumed that angular momentum is effectively transported in the BL, but the transport mechanism is still unknown. Aims. Here we compute detailed model spectra of recently published optically thick one-dimensional radial BL models and qualitatively compare them with observed soft X-ray/extreme ultraviolet (EUV) spectra of dwarf novae in outburst.

Methods. Every considered BL model with given effective temperature and surface density radial distribution is divided into a number of rings, and for each ring, a structure model along the vertical direction is computed using the stellar-atmosphere method. The ring spectra are then combined into a BL spectrum taking Doppler broadening and limb darkening into account.

Results. Two sets of model BL spectra are computed, the first of them consists of BL models with fixed WD mass $\left(1 M_{\odot}\right)$ and various relative WD angular velocities (0.2, 0.4, 0.6 and 0.8 break-up velocities), while the other deals with a fixed relative angular velocity ( 0.8 break-up velocity) and various WD masses $\left(0.8,1\right.$, and $\left.1.2 M_{\odot}\right)$. The model spectra show broad absorption features because of blending of numerous absorption lines, and emission-like features at spectral regions with only a few strong absorption lines. The model spectra are very similar to observed soft X-ray/EUV spectra of SS Cyg and U Gem in outburst. The observed SS Cyg spectrum could be fitted by BL model spectra with WD masses $0.8-1 M_{\odot}$ and relative angular velocities $0.6-0.8$ break up velocities. These BL models also reproduce the observed ratio of BL luminosity and disk luminosity. The difference between the observed and the BL model spectra is similar to a hot optically thin plasma spectrum and could be associated with the spectrum of outflowing plasma with a mass loss rate compatible with the BL mass accretion rate.

Conclusions. The suggested method of computing BL spectra seems very promising and can be applied to other BL models for comparison with EUV spectra of dwarf novae in outburst.
\end{abstract}

Key words. accretion, accretion disks - stars: dwarf novae - radiative transfer - methods: numerical - X-rays: binaries

\section{Introduction}

The importance of energy release between an accretion disk and a central object with a surface was realized almost immediately (Lynden-Bell \& Pringle 1974) after the introduction of modern accretion disk theory (Shakura \& Sunyaev 1973). Later a similar one-dimensional (1D) boundary-layer (BL) theory was developed (Pringle 1977; Pringle \& Savonije 1979; Tylenda 1981; Regev 1983; Bertout \& Regev 1992; Regev \& Bertout 1995; Godon et al. 1995; Popham \& Narayan 1995). Here we mainly consider BLs around white dwarfs (cataclysmic variable stars (CVs), see review in Warner 2003) and describe a few key points of BL theory.

Depending on the accretion rate, $\dot{M}$, a BL can be optically thin $\left(\dot{M}<10^{16} \mathrm{~g} \mathrm{~s}^{-1}\right)$ or optically thick $\left(\dot{M}>10^{16} \mathrm{~g} \mathrm{~s}^{-1}\right)$ (Pringle \& Savonije 1979). The BL optical thickness also depends on the mass and angular velocity of the white dwarf (WD), as well as on the value of turbulent viscosity in the BL (Popham \& Narayan 1995; Collins et al. 2000a). For the description of optically thick BLs, two qualitatively different approaches were suggested, which reduced the problem to a $1 \mathrm{D}$ model. In the first approach, the BL is considered as the inner part of the
1D axi-symmetric accretion disk (Pringle \& Savonije 1979; Regev 1983; Popham \& Narayan 1995) with no vertical component of the velocity. The condition imposed on the BL at the inner boundary is that it rotates at the stellar equatorial velocity. Therefore, the accreting matter has to decelerate and release its excess energy in a relatively narrow (a few percent of the inner disk radius) ring. Most of this energy is radiated away, but part of it can be advected into the WD (Popham 1997; Godon 1997), deposited to the outflow (Mauche \& Raymond 2000), and can accelerate the outer layers of the WD forming a fast rotating belt (Long et al. 2006). In contrast, in the second approach, the matter keeps its nearly Keplerian velocity at the central object's equator and spreads over the surface (Inogamov \& Sunyaev 1999). The spreading matter loses its kinetic energy gradually due to friction with the more slowly rotating surface of the central star and radiates the released energy mainly in two bright high-latitude belts. Initially the spreading layer model was developed for BLs around neutron stars (Inogamov \& Sunyaev 1999), but later it was extended to the WD case, too (Piro \& Bildsten 2004b).

Together with the difference in geometry, the description of the viscosity, which provides a strong coupling of rings of matter 
moving with different velocities and an effective thermal dissipation of the lost energy, is different in both approaches. In the first model the usual $\alpha$ prescription for accretion disks is used, which means that the $r \varphi$-component of the viscosity stress tensor is parameterized by the total pressure $P$ at a given point as $w_{r \varphi}=\alpha P$ (Shakura \& Sunyaev 1973) or, almost equivalently, a similar parametrization of kinematic viscosity, $v_{\alpha}$, is used. In the second approach the $\alpha$ viscosity is completely ignored and only the friction between the high-velocity spreading matter and the dense and relatively cool stellar envelope is considered. The corresponding component of the specific frictional force, $f_{\mathrm{sl}}=\alpha_{\mathrm{b}} \rho_{\mathrm{b}} v_{\mathrm{sl}}^{2}$, is again scaled using the matter density at the bottom of the spreading layer, $\rho_{\mathrm{b}}$, and the relative spreading matter velocity, $v_{\mathrm{sl}}$ (Inogamov \& Sunyaev 1999). The estimated value of $\alpha_{\mathrm{b}}$ is relatively low, about $10^{-3}$ (Inogamov \& Sunyaev 1999). Detailed consideration of this problem shows that the matter deceleration due to friction with the underlying stellar envelope is not trivial and has to be investigated more carefully (Inogamov \& Sunyaev 2010).

It is clear from both viscosity descriptions that we do not know the physics of kinetic energy loss and angular momentum transfer in BLs (see, e.g., Kato \& Inagaki 1994; Narayan et al. 1994; Godon 1995a). The magneto-rotational instability (MRI; Velikhov 1959; Chandrasekhar 1960; Balbus \& Hawley 1991), which is usually considered as a physical realization of the $\alpha$ viscosity, cannot operate in the case of angular velocity decrease taking place in BLs (Godon 1995a). Recently, Belyaev \& Rafikov (2012) and Belyaev et al. (2012, 2013) have suggested a new physical model for an angular momentum transport in BLs based on acoustic instabilities. Various hydrodynamical instabilities, beginning with simple shear instabilities (Kippenhahn \& Thomas 1978), were also considered before as a way of angular momentum transport (see the review in Belyaev \& Rafikov 2012).

Another important feature of BLs is their 3D nature. A full $3 \mathrm{D}$ treatment is important to account for a correct turbulent viscosity description. But BL models that use a parametrization of the turbulent viscosity could be considered as axisymmetric $2 \mathrm{D}$ models. The first attempts to describe the $\mathrm{BL}$ around the WD using a 2D time-dependent hydrodynamical approach were performed many years ago (Robertson \& Frank 1986; Kley \& Hensler 1987; Kley 1989). The importance of the viscosity prescription was also demonstrated (Kley 1991). Recently, this kind of computation was repeated with higher, albeit insufficient temporal and spatial resolution without (Fisker \& Balsara 2005) and with rudimentary radiation treatment (Balsara et al. 2009). These investigations have confirmed that matter is spreading over the WD surface for optically thick BLs.

Astrophysical observations give the possibility to constrain the correct description of angular moment transport and energy dissipation in BLs. It is necessary to compare properties of a mature BL model with observed features. There are two ways for this kind of comparison. The first one is to investigate flux variability, which is probably connected with BLs, using a noise power spectrum approach, for instance van der Klis (1989). There are a lot of observational data about rapid flux variability in low-mass X-ray binaries (LMXBs), such as quasi-periodic oscillations (QPOs) and power density spectra (van der Klis 2000). The cumulative data about rapid variability of CVs, especially in the optical band, is even larger (see, e.g., Patterson 1981; Warner 1986, 2003; Warner \& Pretorius 2008, and references therein). Two types of rapid oscillations were distinguished (Robinson \& Nather 1979), namely high-degree coherent oscillations with relatively short periods $(\sim 7-70 \mathrm{~s})$ in dwarf nova outbursts (DNOs), and less coherent quasi-periodical oscillations (QPOs) with longer periods (up to tens of minutes). The amplitudes of variability for both DNOs and QPOs are relatively low in the optical band $(<0.01 \mathrm{mag})$. On the other hand, the amplitude of these oscillations in X-rays can be much greater (tens of percent, Cordova et al. 1984; Jones \& Watson 1992). It is interesting that the coherent oscillations in SS Cyg are observed in soft X-rays, but not in hard X-rays (Swank 1979; Jones \& Watson 1992). This fact supports the hypothesis that the coherent oscillations might be connected with an optically thick BL that could be responsible for the soft X-ray radiation of SS Cyg during outburst. There are a few relatively simple models to explain DNOs and QPOs (see, e.g., Popham 1999; Warner \& Woudt 2002; Piro \& Bildsten 2004a; Godon 1995b; Collins et al. 1998, 2000b), and part of them are connected with the existing BL models.

The second way is a comparison of observed emergent spectra of close binary systems with predicted spectra of accretion disks and BLs. Emergent spectra could be computed for BL models with the simple local $\alpha$-viscosity, because those models consider energy release and predict some bolometric radiation flux distribution over the BL. BL model spectra in blackbody approximation were computed by many authors (see, e.g. Tylenda 1977; Kley 1991; Popham \& Narayan 1995). The contribution of BL radiation to the FUV spectra of some CVs was also taken into account by computing models of BL rings using the stellar-atmosphere method (Godon \& Sion 2011; Godon et al. 2012).

Luminous LMXBs (with $L>0.05-0.1 L_{\text {Edd }}$ ) with neutron stars show relatively soft and wide X-ray spectra, which can be represented to first approximation by two blackbodies with temperatures $k T$ about $1 \mathrm{keV}$ and $2.5 \mathrm{keV}$ (Mitsuda et al. 1984). It is possible to assume that the first component corresponds to the accretion disk, and the second one corresponds to the BL. Study of X-ray variability helped to distinguish between these two components (Gilfanov et al. 2003; Revnivtsev \& Gilfanov 2006). These authors extracted the spectra of the strongly variable component of the observed X-ray flux in a few LMXBs and found that they are very similar for all the investigated sources and that they can be described by a comptonized plasma spectrum with $k T \approx 2.4 \mathrm{keV}$ and electron scattering optical depth $\tau_{\mathrm{e}} \approx 5-7$. They connected these high-variability components of the X-ray flux to the BL emission. The spectra of these components were described by model spectra of spreading layers (Suleimanov \& Poutanen 2006). Another reference to the validity of the hypothesis that the BLs around neutron stars are spreading layers was presented by Revnivtsev et al. (2013). The high-variability spectral component of the X-ray transient source XTE J1701-462 keeps the same spectral shape when the bolometric luminosity varies by a factor of twenty. The maximum temperature of this component (Wien tail color temperature) is about $2.4-2.6 \mathrm{keV}$ and coincides with the maximum color temperature of the type I X-ray bursts of the same source. It is believed that the latest color temperature of a burst corresponds to the Eddington luminosity (see, e.g., review Lewin et al. 1993). Therefore, most probably, the BLs in LMXBs consist of a part of the neutron star surface that radiates at the Eddington limit with the emitting area being proportional to the luminosity, as it was predicted by the spreading layer model (Inogamov \& Sunyaev 1999).

$\mathrm{X}$-ray and extreme ultraviolet (EUV) radiation of CVs, which can be associated with BL emission, is much more diverse and a univocal interpretation is difficult (see, e.g., Patterson \& Raymond 1985a,b). Blackbody approximation of the optically 
thick BL model spectra predicts a luminous soft X-ray/EUV component with temperature 200-500 kK (Pringle \& Savonije 1979; Popham \& Narayan 1995; Collins et al. 2000a; Piro \& Bildsten 2004b; Hertfelder et al. 2013) in the spectra of all high mass-accretion rate CVs without significant magnetic field (dwarf novae in outburst and nova-like stars). In fact, only a few of them have this kind of component in their spectra, e.g. SS Cyg and U Gem in outburst (Cordova et al. 1984). The X-ray spectra of other non-magnetic CVs are rather hard (van Teeseling \& Verbunt 1994; van Teeseling et al. 1996) and can be described by the model of a cooling flow with temperatures $k T$ from a few $\mathrm{keV}$ up to tens of keV (Done et al. 1995; Mukai et al. 2003; Baskill et al. 2005). This kind of radiation is natural for optically thin BLs (Pringle \& Savonije 1979), or even for the inner hot accretion flow (Revnivtsev et al. 2012) to be expected for dwarf novae in quiescence, but requires some non-trivial explanation for high mass-accreting CVs, like V603 Aql (see, e.g. Patterson \& Raymond 1985b). Formally, Popham \& Narayan (1995) have found that the transition to the optically thin regime in the model BLs could be reached at sufficiently high mass accretion rates. But their results are questionable because they underestimated the Rosseland "true" opacity by at least two orders of magnitude, using Kramers opacity $k_{\mathrm{kr}}=k_{0} \rho T^{-3.5} \mathrm{~cm}^{2} \mathrm{~g}^{-1}$ with $k_{0}=6.6 \times 10^{22}$. The more realistic coefficient is much larger, $k_{0}=5 \times 10^{24}$ (Frank et al. 2002).

At present time, soft X-ray/EUV components were found in four dwarf novae in (super-) outbursts: SS Cyg (Cordova et al. 1980a, 1984; Mauche et al. 1995; Mauche 2004), U Gem (Cordova et al. 1980b, 1984; Long et al. 1996), VW Hyi (Mauche 1996), and OY Car (Mauche \& Raymond 2000). The high-inclination system OY Car shows mainly broad emission lines arising due to resonance scattering in the strong disk wind (Mauche \& Raymond 2000). The high-resolution spectra of the other CVs, obtained by EUVE and Chandra observations, show numerous broad absorption- and emission-like details. These spectra are similar to soft X-ray spectra of supersoft X-ray sources (Lanz et al. 2005; Rauch et al. 2010) and also have to be modeled using stellar model-atmosphere methods. The first attempt to fit the soft X-ray spectrum of SS Cyg in outburst (Mauche 2004) by hot LTE model-atmosphere spectra showed the potential for success of this approach (Suleimanov et al. 2013).

With the work at hand, we start to model the various BL models using the stellar-atmosphere method and a comparison of the results with the properties of observed soft X-ray/EUV spectra of CVs mentioned above. Here we present the model spectra of particular 1D hydrodynamic BL models that were computed recently by Hertfelder et al. (2013).

\section{Method}

Our work is based on the 1D models of BLs between optically thick accretion disks and WDs that were computed in our previous work (Hertfelder et al. 2013). Models are considered in a cylindrical coordinate system $(z, \varphi, R)$, being axisymmetric (independent of $\varphi$ ) and vertically averaged (over $z$-coordinate). They were computed for a fixed mass-accretion rate $\dot{M}=1.5 \times 10^{-8} M_{\odot} \mathrm{yr}^{-1}$, three different values of WD mass, $M_{\mathrm{WD}}=0.8,1$, and $1.2 M_{\odot}$, and five values of WD angular velocity, $\omega_{\mathrm{WD}}=0,0.2,0.4,0.6$ and $0.8 \omega_{\mathrm{K}}$, where $\omega_{\mathrm{K}}$ is the Kepler angular velocity $\omega_{\mathrm{K}}^{2}=G M_{\mathrm{WD}} / R^{3}$ at the WD radius $R_{\mathrm{WD}}$. WD radii were calculated using the Nauenberg (1972) relation. An increase of equatorial radii due to rotation was ignored. A simple $\alpha$ prescription for the viscosity $v_{\alpha}$ was used according to Shakura \& Sunyaev (1973), see details in Hertfelder et al. (2013):

$v_{\alpha}=\alpha a^{2} \omega_{\mathrm{K}}^{-1}$,

where $a$ is the sound speed. We note that $\omega_{\mathrm{K}}$ is taken for the current BL radius, however, the radial extension of model BLs is low and $\omega_{\mathrm{K}}$ for a given model is almost constant. A relatively low value of the $\alpha$ parameter $(\alpha=0.01)$ is assumed for all models in order to avoid supersonic radial motions.

For every 1D model we consider the distribution of the following physical parameters along the radial coordinate $R$ : effective temperature $T_{\text {eff }}(R)$ (or, equivalently, the total radiated flux $\left.F_{0}(R)\right)$, surface density $\Sigma_{0}(R)$, BL half-thickness $H_{0}(R)$, and rotation velocity $v_{\varphi}(R)$. The following steps have to be performed to compute the model emergent spectrum:

- Divide the BL model in a number of rings with equal luminosity.

- Compute a gray model for each ring along the vertical coordinate $z$.

- Starting from the gray model, compute a model of the each ring using model atmosphere methods together with the local emergent spectrum.

- Sum up the local spectra to a total BL spectrum taking the rotation of the rings into account.

This approach is almost identical to the one that was used for the computation of CV accretion disk spectra by many authors (Kriz \& Hubeny 1986; Shaviv \& Wehrse 1991; Suleymanov 1992; Wade \& Hubeny 1998; Nagel et al. 2004). We understand that the local 1D method assumed here is rather crude. But we believe that it gives a reasonable first approximation, which is applicable to comparison with observations. This method is a first step to more sophisticated 2D models.

Accretion disk and BL in the 1D models presented by Hertfelder et al. (2013) are considered as a comprehensive structure, and the boundary between accretion disk and BL is blurred. However, the $T_{\text {eff }}(R)$ distributions have a local minimum at $R \approx 1.1 R_{\mathrm{WD}}$. We adopted that radius as the boundary between the disk and the BL and studied the BLs at radii lesser than this boundary. We divided each BL model into a few (5-20) rings, and the particular radius $R_{j}$ of a given ring with number $j$, for which a local vertical structure and local emergent spectrum are computed, is determined by the condition

$F_{0}\left(R_{j}\right) \Delta R_{j}=F_{0}\left(R_{j}\right)\left(R_{j}^{\mathrm{U}}-R_{j}^{\mathrm{L}}\right)=\int_{R_{j}^{\mathrm{L}}}^{R_{j}^{\mathrm{U}}} F_{0}(R) \mathrm{d} R$,

where $R_{j}^{\mathrm{U}}$ and $R_{j}^{\mathrm{L}}$ are the radii of the upper and lower ring boundaries, respectively. We note that $R_{j-1}^{\mathrm{U}}=R_{j}^{\mathrm{L}}$. detail.

We now describe the other steps of our method in more

\subsection{BL vertical structure. Gray atmosphere approach}

The approach employed here is based on the numerical method used for modeling of accretion disk structures over the $z$ coordinate as presented by Suleimanov et al. (2007 - see also Kriz \& Hubeny 1986; Shaviv \& Wehrse 1991; Suleymanov 1992; Wade \& Hubeny 1998; Nagel et al. 2004). Some modifications are made and described additionally.

The vertical structure of each $\mathrm{BL}$ ring at radius $R_{j}$ is determined by the ring parameters $T_{\text {eff }}, \Sigma_{0}, H_{0}$, and a set of differential 
equations. The first one is the hydrostatic equilibrium equation

$\frac{1}{\rho} \frac{\mathrm{d} P}{\mathrm{~d} z^{\prime}}=g_{z}=\frac{\left(z_{1}-z^{\prime}\right)}{\left(1+\left(z_{1}-z^{\prime}\right)^{2} / R_{j}^{2}\right)^{1 / 2}} \omega_{\mathrm{K}}^{2}\left(R_{j}\right)$,

where $P=P_{\mathrm{g}}+P_{\text {rad }}$ is the total pressure, the sum of gas and radiation pressure, $\rho$ is the matter density, $z_{1}$ is a parameter, the distance from the mid-plane to the highest point of the vertical model, and $z^{\prime}=z_{1}-z$ is the vertical coordinate, which is equal 0 at the highest point of the model.

The second equation is the energy-conservation law. The correct local energy generation rate $\mathrm{d} F / \mathrm{d} z$ in accretion disks as well as in BLs is not known and a local version of the $\alpha$ approach is often used (see e.g. Suleimanov et al. 2007). Fortunately, local emergent spectra of optically thick accretion disks (and, therefore, the BL models considered here, too) are weakly dependent on details of the energy generation rate, so we took the simplest version (Shakura \& Sunyaev 1973):

$\frac{1}{\rho} \frac{\mathrm{d} F}{\mathrm{~d} z^{\prime}}=-2 \frac{F_{0}}{\Sigma_{0}}$

Here $F$ is the integral (bolometric) vertical flux at given height, $F_{0}=\sigma_{\mathrm{SB}} T_{\text {eff }}^{4}$, is the emergent integral flux. The energy conservation law has the integral (Shakura \& Sunyaev 1973)

$F(m)=F_{0}\left(1-\frac{2 m}{\Sigma_{0}}\right)$

where the boundary condition $F(m=0)=F_{0}$ is used. Here the Lagrangian coordinate $m$ is determined by the equation

$\mathrm{d} m=\rho \mathrm{d} z^{\prime}$,

and, therefore,

$\Sigma_{0}=\int_{-\infty}^{+\infty} \mathrm{d} m \approx 2 \int_{0}^{z_{1}} \rho \mathrm{d} z^{\prime}$.

We assumed a purely radiative transport of energy in $z$-direction, therefore, the third equation is the radiation transfer equation. For the gray approach we use the first moment of that equation

$\frac{1}{\rho} \frac{\mathrm{d} P_{\mathrm{rad}}}{\mathrm{d} z^{\prime}}=\frac{\kappa_{\mathrm{R}} F}{c}$

where $\kappa_{\mathrm{R}}$ is the Rosseland opacity, which is determined as the greater value of electron scattering, $\sigma_{\mathrm{e}}=0.335 \mathrm{~cm}^{2} \mathrm{~g}^{-1}$, and Kramers opacity, $\kappa_{\mathrm{kr}}=5 \times 10^{24} \rho T^{-3.5}$. The sum of these values is also relevant (Popham \& Narayan 1995).

These equations are solved together with the ideal gas law

$P_{\mathrm{g}}=n k T$,

where $n$ is the total number density of particles. We assume full local thermodynamic equilibrium (LTE). Therefore, the local gas temperature $T$ can be found from the integrated (over frequency) mean intensity $J$

$J=\frac{3 c P_{\mathrm{rad}}}{4 \pi}=B(T)=\frac{\sigma_{\mathrm{SB}} T^{4}}{\pi}$.

Here we used the following TE relations between radiation energy density $\varepsilon_{\text {rad }}$, integral mean intensity $J$, and radiation pressure:

$\varepsilon_{\mathrm{rad}}=\frac{4 \pi J}{c}=3 P_{\mathrm{rad}}$.

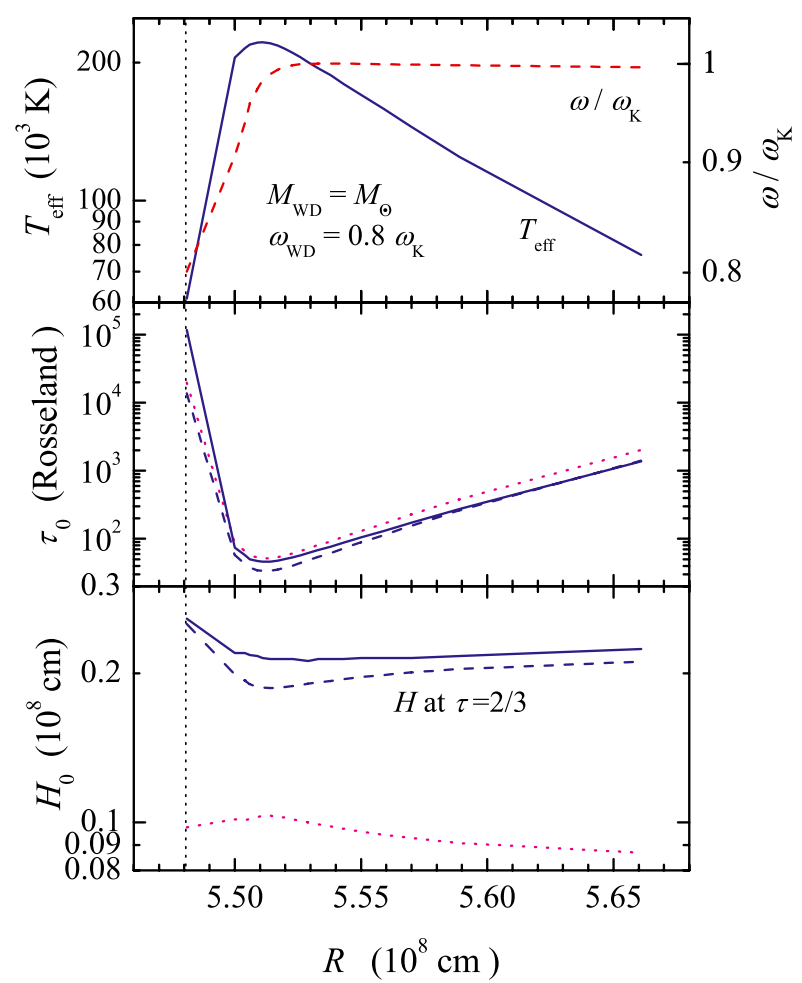

Fig. 1. Distribution of BL quantities along radius. The data are from the model with $M_{\mathrm{WD}}=M_{\odot}$ and $\omega_{\mathrm{WD}}=0.8 \omega_{\mathrm{K}}$. The position of the WD surface at $R_{\mathrm{WD}}=5.48 \times 10^{8} \mathrm{~cm}$ is shown by the vertical dotted line. Top panel: shown is the effective temperature (solid curve) together with the relative angular velocity (dashed curve), as obtained from the $1 \mathrm{D}$ hydrodynamical model. These are the same for the atmosphere models. Middle and bottom panels: Rosseland optical half-thickness, $\tau_{0}$, and geometrical half-thickness, $H_{0}$, for the model-atmosphere models (solid lines). The distributions obtained from the 1D hydrodynamical model (dotted curves) and the gray model (dashed curves) are also shown.

A solar chemical composition is assumed and all ionization states of the 15 most abundant chemical elements are taken into account. The relation between density $\rho$, total number density $n$, and electron number density $n_{\mathrm{e}}$ is found using Saha's equation for each considered ionization state.

We solve Eqs. (3) and (5-8) from the surface $z^{\prime}=0$ to the mid-plane $z^{\prime}=z_{1}$ by using a shooting method with the boundary conditions

$F(0)=F_{0}, \quad \rho(0)=0, \quad P(0)=P_{\mathrm{rad}}(0)=\frac{2}{3} \frac{F_{0}}{c}$.

There is one additional parameter unknown at the outset: $z_{1}$. We find it by the dichotomy method on the range (1-30) $H_{0}$ using an additional boundary condition at the mid-plane

$F\left(z^{\prime}=z_{1}\right)=0$

or, equivalently,

$m\left(z^{\prime}=z_{1}\right)=\frac{\Sigma_{0}}{2}$.

A comparison of optical depths and half-thicknesses of the 1D and the gray-approach models along $z$ coordinate for one particular BL model $\left(M_{\mathrm{WD}}=M_{\odot}\right.$ and $\left.\omega_{\mathrm{WD}}=0.8 \omega_{\mathrm{K}}\right)$ is shown in Fig. 1 in the two bottom panels. It is clear that the new halfthickness of the BL model $H=z_{1}-z\left(\tau_{\mathrm{R}}=2 / 3\right)$ is approximately twice as large as the thickness of the $1 \mathrm{D}$ hydrodynamic 
Table 1. Parameters of the rings for the models with parameters $M_{\mathrm{WD}}=M_{\odot}$ and $\omega_{\mathrm{WD}}=0.8,0.6,0.4,0.2 \omega_{\mathrm{K}}$.

\begin{tabular}{llllll}
\hline \hline$R, 10^{8} \mathrm{~cm}$ & $T_{\text {eff }}, 10^{3} \mathrm{~K}$ & $\Sigma_{0}, \mathrm{~g} / \mathrm{cm}^{2}$ & $H, 10^{7} \mathrm{~cm}$ & $\tau_{0}$ & $\omega / \omega_{\mathrm{K}}$ \\
\hline 5.493 & 154.4 & 499.6 & 2.188 & 230 & 0.839 \\
5.513 & 219.6 & 140.4 & 1.866 & 34.1 & 0.987 \\
5.522 & 209.4 & 152.9 & 1.882 & 40.3 & 0.998 \\
5.543 & 180.3 & 207.1 & 1.945 & 71.1 & 1.000 \\
5.604 & 109.5 & 466.8 & 2.059 & 404 & 0.998 \\
& & & & & \\
5.482 & 241.2 & 376 & 2.36 & 79.8 & 0.652 \\
5.505 & 298.0 & 83.4 & 1.938 & 13.9 & 0.999 \\
5.515 & 283.2 & 93.5 & 1.889 & 15.7 & 1.003 \\
5.534 & 253.8 & 121.1 & 1.905 & 23.1 & 1.002 \\
5.610 & 144.1 & 363.1 & 2.094 & 187 & 0.999 \\
& & & & & \\
5.479 & 302.6 & 306 & 2.565 & 51.2 & 0.475 \\
5.499 & 356.6 & 68.3 & 2.395 & 11.4 & 0.994 \\
5.512 & 333.1 & 75.7 & 2.205 & 12.7 & 1.005 \\
5.538 & 302.9 & 101 & 2.089 & 16.9 & 1.004 \\
5.623 & 170.1 & 330 & 2.145 & 125 & 0.999 \\
& & & & & \\
5.478 & 349.7 & 264 & 2.998 & 44.1 & 0.299 \\
5.498 & 410.0 & 62 & 3.115 & 10.3 & 0.992 \\
5.515 & 372.7 & 69.3 & 2.619 & 11.6 & 1.006 \\
5.540 & 331.5 & 85.2 & 2.272 & 14.2 & 1.004 \\
5.708 & 188.9 & 648 & 2.554 & 203 & 0.997 \\
\hline
\end{tabular}

Table 2. Parameters of the rings for the models with parameters $\omega_{\mathrm{WD}}=0.8 \omega_{\mathrm{K}}$ and $M_{\mathrm{WD}}=1.2$ and $0.8 M_{\odot}$.

\begin{tabular}{llllll}
\hline \hline$R, 10^{8} \mathrm{~cm}$ & $T_{\text {eff }}, 10^{3} \mathrm{~K}$ & $\Sigma_{0}, \mathrm{~g} / \mathrm{cm}^{2}$ & $H, 10^{7} \mathrm{~cm}$ & $\tau_{0}$ & $\omega / \omega_{\mathrm{K}}$ \\
\hline 3.865 & 222.2 & 565.6 & 1.370 & 179 & 0.829 \\
3.873 & 304.3 & 164.7 & 1.187 & 30.6 & 0.961 \\
3.886 & 284.1 & 176.7 & 1.180 & 36.5 & 0.993 \\
3.895 & 253.0 & 227.2 & 1.204 & 56.9 & 0.993 \\
3.936 & 150.5 & 559.9 & 1.275 & 356 & 0.991 \\
& & & & & \\
7.044 & 113.8 & 461.3 & 3.166 & 299 & 0.848 \\
7.065 & 167.9 & 147.7 & 2.784 & 46.2 & 0.965 \\
7.092 & 156.8 & 156.0 & 2.788 & 55.1 & 1.002 \\
7.114 & 139.5 & 192.0 & 2.850 & 83.9 & 1.004 \\
7.205 & 84.37 & 426.8 & 3.026 & 482 & 1.001 \\
\hline
\end{tabular}

model (red dotted line). This is a well known factor (Shakura \& Sunyaev 1973) because the half-thickness of 1D models is just the pressure scale height $H_{0}=a \omega_{\mathrm{K}}^{-1}$, while the new thickness is given by the height of the photosphere. The effective temperatures are plotted in the top panel of Fig. 1, here the models agree by definition. The gray temperature structure of the hottest ring of the same BL model is shown in Fig. 2 (bottom panel). We note that this ring is a reference model for our illustration of the presented method. The parameters of the rings for all computed BL models with five rings are shown in Tables $1-3$. The ring half-thicknesses, $H$, and the ring Rosseland optical depths, $\tau_{0}=\int_{0}^{z_{1}} k_{\mathrm{R}} \rho \mathrm{d} z$, were computed by using the gray ring models.

Every computed gray vertical ring model is interpolated to a logarithmically equidistant column density grid with 98 depth points in the range from $\sim 10^{-7} \mathrm{~g} \mathrm{~cm}^{-2}$ to $\Sigma_{0} / 2$. This interpolated ring model is used as a starting model for the calculation of a non-gray model and its emergent spectrum.
Table 3. Parameters of the rings for the model with parameters $M_{\mathrm{WD}}=0.8 M_{\odot}$ and $\omega_{\mathrm{WD}}=0.6 \omega_{\mathrm{K}}$.

\begin{tabular}{llllll}
\hline \hline$R, 10^{8} \mathrm{~cm}$ & $T_{\text {eff }}, 10^{3} \mathrm{~K}$ & $\Sigma_{0}, \mathrm{~g} / \mathrm{cm}^{2}$ & $H, 10^{7} \mathrm{~cm}$ & $\tau_{0}$ & $\omega / \omega_{\mathrm{K}}$ \\
\hline 7.022 & 177.7 & 297 & 3.247 & 85.5 & 0.670 \\
7.044 & 233.3 & 78.6 & 2.631 & 14.0 & 0.972 \\
7.069 & 222.0 & 83.1 & 2.628 & 16.0 & 1.007 \\
7.094 & 196.0 & 115 & 2.751 & 27.2 & 1.006 \\
7.200 & 111.3 & 323 & 3.050 & 215 & 1.002 \\
\hline
\end{tabular}
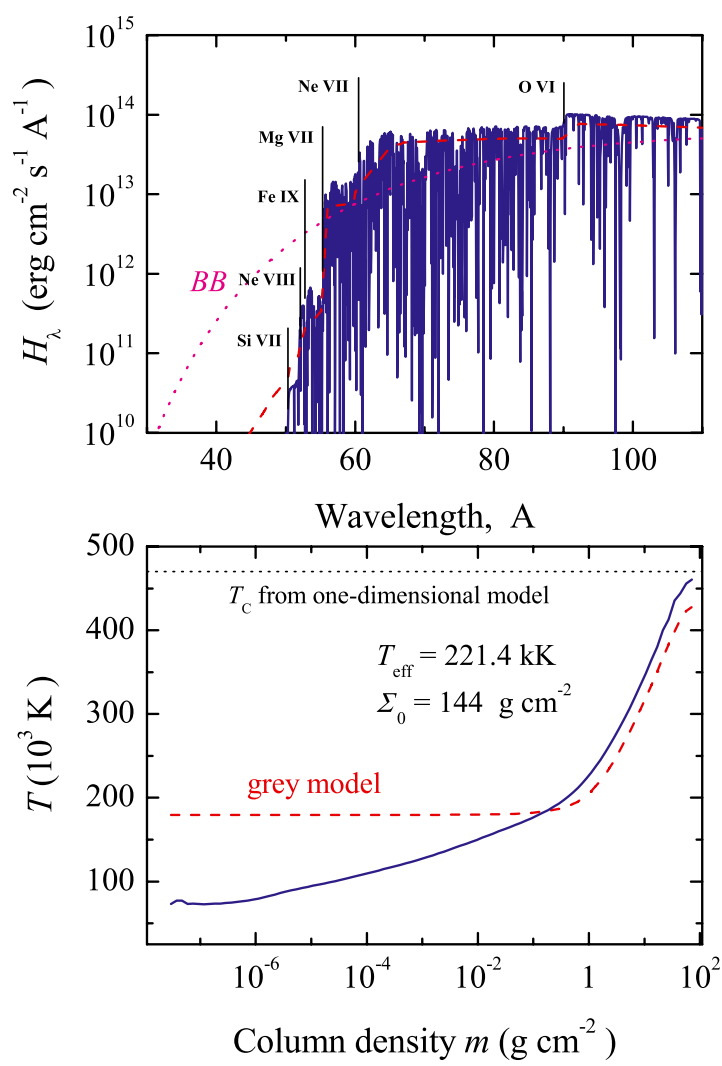

Fig. 2. Top panel: local spectra of the hottest ring of the same BL model as in Fig. 1 computed with (solid curve) and without (dashed curve) spectral lines taken into account. The blackbody spectrum corresponding to the effective temperature is shown by the dotted curve. Bottom panel: temperature structures of the model with lines (solid curve) and the gray model (dashed curve). The central temperature of the hydrodynamical $1 \mathrm{D}$ model is shown by the dotted line.

\subsection{Boundary layer vertical structure. Model atmosphere approach}

The non-gray model is determined by Eqs. (3) and (5), rewritten in the form

$\frac{\mathrm{d} P_{\mathrm{g}}}{\mathrm{d} m}=g_{z}-g_{\mathrm{rad}}$,

where $g_{z}$ and $g_{\text {rad }}$ are defined by Eqs. (3) and (20). Here we again assume that energy is transferred by radiation alone and this is described by the radiation transfer equation at every considered frequency point

$\mu \frac{\mathrm{d} I_{v}}{\mathrm{~d} \tau_{v}}=I_{v}-S_{v}$,

where the monochromatic optical depth is determined from

$\mathrm{d} \tau_{v}=\left(\sigma_{\mathrm{e}}+k_{v}\right) \mathrm{d} m$, 


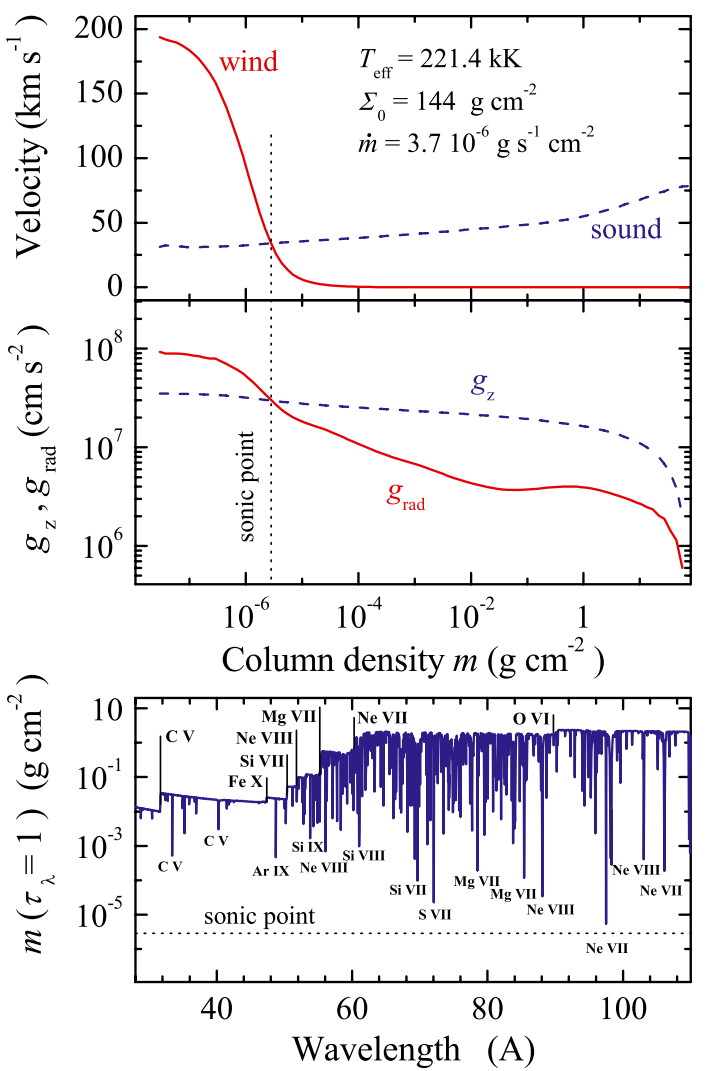

Fig. 3. Top panel: sound speed (dashed curve) and wind velocity (solid curve) distributions along depth in the ring model shown in Fig. 2. Middle panel: distributions of gravity (dashed curve) and radiative acceleration (solid curve) along depth. The position of the sonic point is marked by the dotted vertical line. Bottom panel: depths where the emergent spectrum forms $\left(\tau_{\lambda}=1\right)$. The position of the sonic point is marked by the dashed horizontal line.

and the source function can be expressed as

$S_{v}=\frac{k_{v}}{\sigma_{\mathrm{e}}+k_{v}} B_{v}+\frac{\sigma_{\mathrm{e}}}{\sigma_{\mathrm{e}}+k_{v}} J_{v}$

Here $\mu=\cos \theta$ is the cosine of angle $\theta$, which is the angle between the radiation transfer direction and the ring normal. Eq. (16) determines the specific intensity $I_{v}$ at given frequency and $\mu$. The mean intensity $J_{v}$ and Eddington flux $H_{v}=F_{v} / 4 \pi$ are defined by

$J_{v}=\frac{1}{2} \int_{-1}^{+1} I_{v} \mathrm{~d} \mu, \quad H_{v}=\frac{1}{2} \int_{-1}^{+1} \mu I_{v} \mathrm{~d} \mu$.

We took coherent electron scattering $\sigma_{\mathrm{e}}$ into account together with the free-free and bound-free opacities $k_{v}$ of all ions of the 15 most abundant elements using opacities from Verner \& Yakovlev (1995) and Verner et al. (1996). Line blanketing was taken into account using 25000 spectral lines from the CHIANTI, Version 3.0, atomic database (Dere et al. 1997).

We solve the radiation transfer equation (16) at three values of $|\mu|$ using the short characteristics method (Olson \& Kunasz 1987). The adopted $\mu$ values correspond to Chebyshev-Gauss quadrature abscissas to accurately compute the integrals in (19).

Radiative acceleration $g_{\text {rad }}$ is determined by

$g_{\mathrm{rad}}=\frac{4 \pi}{c} \int_{0}^{\infty}\left[\sigma_{\mathrm{e}}+k_{v}\right] H_{v}(m) \mathrm{d} v$
A correct model has to satisfy the energy balance equation which can be written in two forms:

$4 \pi \int_{0}^{\infty} H_{v}(m) \mathrm{d} v=F_{0}\left(1-\frac{2 m}{\Sigma_{0}}\right)$

and

$4 \pi \int_{0}^{\infty}\left[\sigma_{\mathrm{e}}+k_{v}\right]\left[J_{v}-S_{v}\right] \mathrm{d} v=-\frac{2 F_{0}}{\Sigma_{0}}$.

Of course, the initial model does not satisfy the energy balance equation, having the relative flux error

$\varepsilon_{\mathrm{H}}(m)=1-\frac{F_{0}\left(1-2 m / \Sigma_{0}\right)}{4 \pi \int_{0}^{\infty} H_{v}(m) \mathrm{d} v}$

and flux derivative error

$\varepsilon_{\Lambda}(m)=4 \pi \int_{0}^{\infty}\left[\sigma_{\mathrm{e}}+k_{v}\right]\left[J_{v}-S_{v}\right] \mathrm{d} v+\frac{2 F_{0}}{\Sigma_{0}}$,

at each depth. It is possible to find corrections to the temperature $\Delta T(m)$ using two methods modified for a non-constant bolometric flux over the ring depth (Suleymanov 1992): the integral $\Lambda$-iteration together with a surface temperature correction for the optically thin parts of the atmosphere

$\Delta T_{\Lambda}(m)=-\varepsilon_{\Lambda}(m)\left(\int_{0}^{\infty}\left[\frac{\Lambda_{v, \text { diag }}-1}{1-\alpha_{v} \Lambda_{v, \text { diag }}}\right] k_{v} \frac{\mathrm{d} B_{v}}{\mathrm{~d} T} \mathrm{~d} v\right)^{-1}$,

where $\alpha_{v}=\sigma_{\mathrm{e}} /\left(k_{v}+\sigma_{\mathrm{e}}\right)$, and $\Lambda_{v \text {, diag }}$ is the diagonal matrix element of the $\Lambda$-operator, and the Avrett-Krook flux correction based on the relative flux error $\varepsilon_{\mathrm{H}}(m)$ for the optically thick parts of the ring model

$\Delta T_{\mathrm{H}}(m)=-\frac{\mathrm{d} T}{\mathrm{~d} m} \int_{0}^{m} \varepsilon_{\mathrm{H}}(x) \mathrm{d} x$.

Both methods were described in detail by Kurucz (1970).

Then we find new values of the gas pressure using new $g_{\mathrm{rad}}$ and recalculate the densities. Subsequently, opacities are recalculated and the radiation transfer equations at all frequencies are resolved and a new temperature structure is computed. Then we recalculate the geometrical depth scale $z^{\prime}(m)$ using the new densities. This procedure is performed up to convergence using our version of the computer code ATLAS (Kurucz 1970) that was modified to deal with high temperatures (Ibragimov et al. 2003; Suleimanov \& Werner 2007; Suleimanov et al. 2013). We assumed LTE and accounted for pressure ionization effects using the occupation probability formalism (Hummer \& Mihalas 1988), as described by Hubeny et al. (1994).

Because of the significant effect of spectral lines on the radiation force $g_{\mathrm{rad}}$, the atmosphere modeling approach is divided into two steps. In the first step we compute a continuum model without spectral lines, which provides the radiation force $g_{\mathrm{rad}}<g_{z}$ at all BL ring depths. The distributions of basic BL quantities for continuum ring models are presented in Fig. 1 by solid curves. Hydrostatic ring models cannot be computed when spectral lines are included, because then the radiation force $g_{\text {rad }}$ becomes higher than the gravity $g_{z}$ in the upper, optically thin (in continuum) layers of the models. Therefore, a line-driven wind has to arise from the BL surface, which is similar to the linedriven winds from hot stars (Castor et al. 1975; Kudritzki \& Puls 2000). This kind of wind also exists for CV accretion disks (Drew \& Verbunt 1985; Prinja \& Rosen 1995; Proga et al. 1998). 


\subsubsection{Line-driven wind approach}

Here, we are not interested in the properties of the wind itself, because it probably effects only the strongest spectral lines but not the continuum or moderate and weak spectral lines. In previous works (Ibragimov et al. 2003; Suleimanov et al. 2013), where hot WD model atmospheres were computed, the gas pressure was artificially fixed to $10 \%$ of the total pressure at the upper layers, where $g_{\mathrm{rad}}>g_{z}$. The condition $P_{\text {gas }}=0.1 P$ was chosen because it was approximately correct at those atmospheric layers, where $g_{\text {rad }} \approx g_{z}$. This condition physically means that we assume some artificial wind velocity law that satisfies the imposed condition. Unfortunately, this simple approach is not working for the BL case because gravity $g_{z}$ depends on the vertical coordinate $z$ (it is constant for ordinary stellar atmospheres). Therefore, we develop another simple hydrodynamic approach which takes into consideration an expansion of the upper ring layers. This approach is not self-consistent as we do not include effects of motion in the radiation transfer. Thus we consider a hydrodynamical model for the ring upper layers but the radiation pressure force governing this expansion is computed for a formally static medium. This approach allows computing the model emergent spectra but the obtained wind properties such as the local mass-loss rate and wind-velocity distributions are not completely correct and cannot be considered as reliable results.

The considered approach is based on Euler's equation

$\frac{\mathrm{d}}{\mathrm{d} z}\left(\dot{m} v+P_{\mathrm{g}}\right)=-\rho\left(g_{z}-g_{\mathrm{rad}}\right)$

where $\dot{m}$ is the local mass-loss rate $\left([\dot{m}]=\mathrm{g} \mathrm{s}^{-1} \mathrm{~cm}^{-2}\right)$ and $v$ is the gas velocity at given $z$. This equation can be rewritten as

$\dot{m}\left(1-\frac{a^{2}}{v^{2}}\right) \frac{\mathrm{d} v}{\mathrm{~d} z}=-\rho\left(g_{z}-g_{\mathrm{rad}}\right)-\rho \frac{\mathrm{d} a^{2}}{\mathrm{~d} z}$,

where $a$ is the sound speed connected with gas pressure and matter density by

$P_{\mathrm{g}}=\rho a^{2}$.

Here we used the continuity equation for the plane 1D motion

$\dot{m}=\rho v$.

It is well known (see, e.g. Mihalas 1978) that a correct solution of Eq. (28) has to pass through a singular point with $|v|=a$, where the left and the right side of the equation vanish simultaneously. Therefore, these two conditions must be fulfilled in the singular point:

$a^{2}=v^{2}$

and

$\frac{\mathrm{d} a^{2}}{\mathrm{~d} z}=-\left(g_{z}-g_{\mathrm{rad}}\right)$

or

$C=\frac{\mathrm{d} a^{2}}{\mathrm{~d} z}+\left(g_{z}-g_{\mathrm{rad}}\right)=0$.

We suggest the following scheme to compute a model of the $\mathrm{BL}$ ring with a line-driven wind. We take the model computed without lines as an initial model and solve the radiation-transfer equation with spectral lines taken into account. As a result we obtain that the radiative acceleration $g_{\text {rad }}$ is greater than the current gravity at the upper layers. At this stage we have the gas pressure $P_{\mathrm{g}}(m)$ and the gas density $\rho(m)$ distributions from the continuum model, and the radiative acceleration $g_{\mathrm{rad}}(m)$ and the gas temperature $T(m)$ distributions after the first temperaturecorrection iteration. The geometrical depth scale $z(m)$ and corresponding gravity $g_{\mathrm{z}}$ are connected with the gas density of the initial (continuum) model. Using these distributions we can find the sound-speed distribution $a(m)$ and its derivative $\mathrm{d} a(m) / \mathrm{d} z$, and then find the depth $m_{\mathrm{c}}$ where Eq. (32) is satisfied. We know at this depth the wind velocity must be equal to the sound speed $a\left(m_{\mathrm{c}}\right)$. Therefore, we can evaluate the local mass-loss rate

$\dot{m}=\rho\left(m_{\mathrm{c}}\right) a\left(m_{\mathrm{c}}\right) / 2$

using the continuity Eq. (30). Here we have taken into account that the correct gas density $\rho_{\mathrm{w}}$ in the singular point for the model with the wind must be half of that in the hydrostatic model $\rho_{\mathrm{st}}$, see Eq. (27)

$\dot{m} a+\rho_{\mathrm{w}} a^{2}=\rho_{\mathrm{w}} a^{2}+\rho_{\mathrm{w}} a^{2}=\rho_{\mathrm{st}} a^{2}$.

Using this mass-loss rate $\dot{m}$ we solve two ordinary differential equations

$\frac{\mathrm{d} P_{\mathrm{w}}}{\mathrm{d} z}=-\rho\left(g_{z}-g_{\mathrm{rad}}\right)$

and

$\frac{\mathrm{d} m^{\prime}}{\mathrm{d} z}=\rho$,

where $P_{\mathrm{w}}=\dot{m} v+\rho a^{2}$, on a new fine and equidistant geometrical depth grid $z$ (50000 points) from the midplane $(z=0)$ up to the surface $\left(m^{\prime}=\Sigma_{0} / 2\right)$ with the boundary conditions $v=0$, $m^{\prime}=0$, and $P_{\mathrm{w}}(0), \rho(0)=P_{\mathrm{w}}(0) / a(0)^{2}$ at the midplane, using the shooting method. The current gas density is calculated using the current $P_{\mathrm{w}}$

$\rho=\frac{P_{\mathrm{w}}}{2 a^{2}} \pm \sqrt{\left(\frac{P_{\mathrm{w}}}{2 a^{2}}\right)^{2}-\left(\frac{\dot{m}}{a}\right)^{2}}$.

The plus sign is used for wind velocities below the sonic point and the minus sign in the supersonic part of the model. The wind velocity is found using the continuity Eq. (30). The necessary current values of the $g_{\mathrm{rad}}\left(m^{\prime}\right)$ and $\mathrm{d} a^{2}\left(m^{\prime}\right) / \mathrm{d} z=\rho \mathrm{d} a^{2}\left(m^{\prime}\right) / \mathrm{d} m^{\prime}$ are found using a spline interpolation in the dependencies $g_{\text {rad }}(m)$ and $\mathrm{d} a^{2}(m) / \mathrm{d} m$ known from the previous iteration, taking $m=\Sigma_{0} / 2-m^{\prime}$.

Usually, the necessary conditions Eqs. (28) and (32) are not satisfied at the sonic point for the first tried initial condition for $P_{\mathrm{w}}(0)$. Therefore, we find the necessary $P_{\mathrm{w}}(0)$ with the dichotomy method. Usually, the correct initial value of the gas pressure at the midplane differs by a few percent from the gas pressure at the midplane found in the previous iteration. We adopt the two following numerical convergence criteria at the singular point

$\frac{a-|v|}{a}<10^{-2}, \quad \frac{C}{g_{z}}<10^{-2}$.

After obtaining the solution, all quantities are interpolated on the model atmosphere column density grid $m$ (98 points) which is used for the radiation transfer solution. Therefore, we have 
a new distribution of the gas pressure $P_{\mathrm{g}}=\rho a^{2}$ with the linedriven wind taken into account which is in accordance with the radiative acceleration $g_{\text {rad }}$. Then we find a new gas density distribution using the new $P_{\mathrm{g}}$ and the temperature distribution obtained after the temperature-correction iteration. For this aim the ideal gas Eq. (9) is used together with charge and number density conservation laws. Then the radiation transfer is re-solved and a new temperature correction is performed. The above described procedure to find a new gas-pressure distribution is repeated with a new radiative-acceleration distribution. There is only one difference. The local mass-loss rate is now calculated (see Eq. (34)) without division by 2 because the current model takes into consideration the line-driven wind.

This iteration scheme is converging and it is stable. As a result we get a self-consistent model of the BL ring and its emergent spectrum. Results of calculations for the reference ring model are presented in Figs. 2 and 3. The emergent spectrum (Fig. 2, top panel) is similar to hot stellar atmospheres with similar effective temperatures (see e.g. Suleimanov et al. 2013) and shows the same absorption edges as the continuum spectrum together with a forest of spectral absorption lines. The temperature structure (Fig. 2, bottom panel) differs significantly from the gray temperature distribution mainly because of line-blanketing effects. The final model's wind velocity law (Fig. 3, top panel) and the radiation force $g_{\mathrm{rad}}$ distribution (Fig. 3, middle panel) are shown together with the sound speed $a$ and gravity $g_{z}$ distributions. The sonic point is seen to be located at the upper ring layers $\left(m \approx 10^{-5} \mathrm{~g} \mathrm{~cm}^{-2}\right.$ ) and the corresponding mass-loss rate is sufficiently low $\left(\dot{m} \approx 10^{-5}-10^{-6} \mathrm{~g} \mathrm{~s}^{-1} \mathrm{~cm}^{-2}\right)$. Formally, the sonic point is above the formation depths of even the strongest spectral lines (Fig. 3, bottom panel) and, therefore, the wind does not affect the spectrum.

\subsection{Integral BL spectra}

When the computation of all ring model spectra is finished, we integrate them over the BL radial coordinate with taken Doppler broadening due to ring rotation into account

$$
\begin{aligned}
L_{\lambda} & =\cos i \int_{\mathrm{R}_{\text {in }}}^{R_{\text {out }}} R \mathrm{~d} R \int_{0}^{\pi} I_{\lambda^{\prime}}(\cos i) \mathrm{d} \varphi \\
& \approx \cos i \sum_{\mathrm{j}=1}^{\mathrm{N}_{\mathrm{R}}} R_{\mathrm{j}} \Delta R_{\mathrm{j}} \sum_{\mathrm{k}=1}^{\mathrm{N}_{\varphi}} \Delta \varphi_{\mathrm{k}} I_{\lambda^{\prime}}(\cos i),
\end{aligned}
$$

where $i$ is the inclination angle of the BL relative to the line of sight, $I_{\lambda}(\cos i)$ is the local specific intensity in the direction of the line of sight, $N_{\mathrm{R}}$ is the number of considered BL rings (5 or 20 ), and $N_{\varphi}=100$ is a number of considered ring sectors. Here $\lambda^{\prime}$ is the Doppler shifted wavelength:

$\lambda^{\prime}=\lambda+\frac{v_{\varphi}(R)}{c} \sin i \cos \varphi$

The azimuthal velocity distribution $v_{\varphi}(R)$ is taken from the 1D $\mathrm{BL}$ model. We note that integration is only performed over the visible BL part. We assume that we see only a quarter of the total BL surface, which is situated in front of the WD on the visible part of the disk, and that the visible part does not depend on the inclination angle. The change of the projection area of the BL's visible part is proportional to cos $i$. Specific intensities for every ring model are computed using Eq. (16) for six angles relative to the normal: $0,15,30,45,60$ and 75 degrees. Therefore, integral spectra can be computed for these inclination angles relative to the line of sight. We ignored any relativistic effects because they are low.
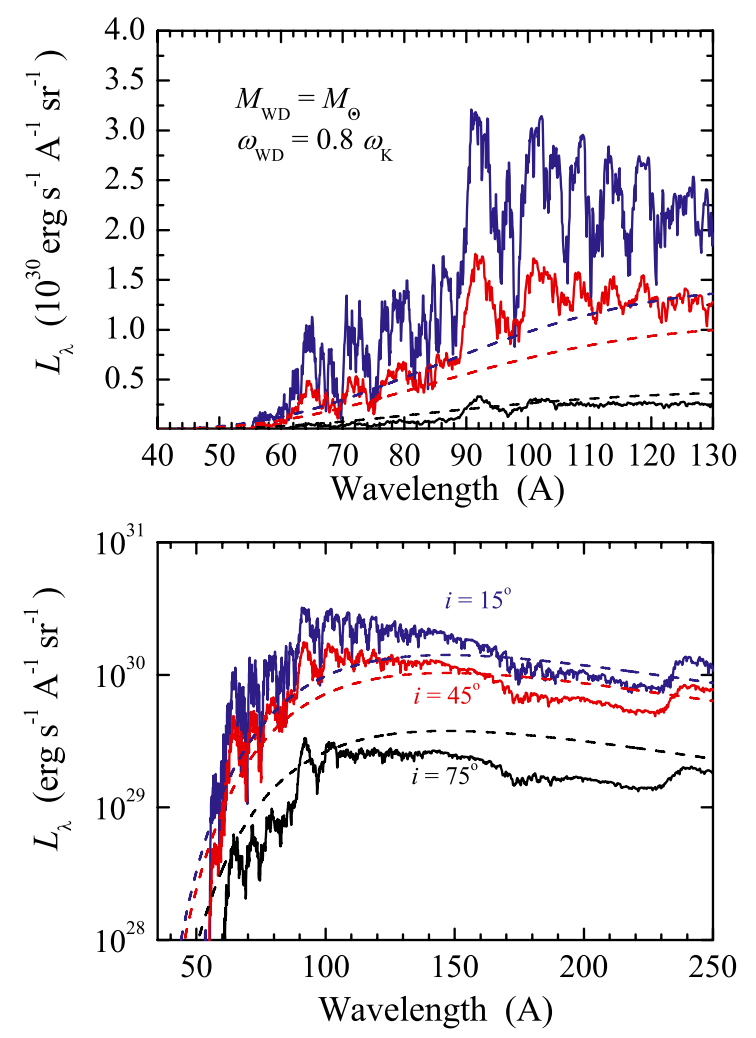

Fig. 4. Spectra of the BL model $M=M_{\odot}, \omega_{\mathrm{WD}}=0.8 \omega_{\mathrm{K}}$ for three inclination angles relative to the line of sight. The corresponding blackbody approximations are shown by dashed curves.

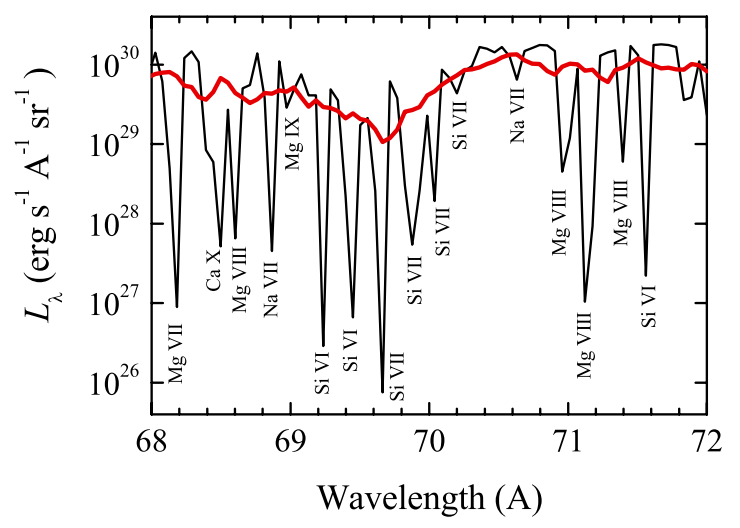

Fig. 5. Detail of the integral emergent fluxes of the same BL model as in Fig. 4 computed for $i=0^{\circ}$ (rotation does not affect the spectrum, thin black curve), and $15^{\circ}$ (thick red curve).

\section{Results}

Using the method presented above, we computed spectra of several BL models. Examples of the integral reference BL model spectra for three angles are shown in Fig. 4. These spectra are calculated using the BL model partitioned in 20 rings. The spectra show broad absorption and quasi-emission features. The absorption features arise due to rotational broadening and blending as a result of many absorption lines at this place of the spectrum. The quasi-emission features are continuum sections with a low amount of absorption lines. These statements are illustrated by Fig. 5, where a narrow section of the integral spectra computed for $i=0^{\circ}$ and $15^{\circ}$ are shown. In Fig. 4 the spectra computed in blackbody approximation are also shown. We focus attention on 

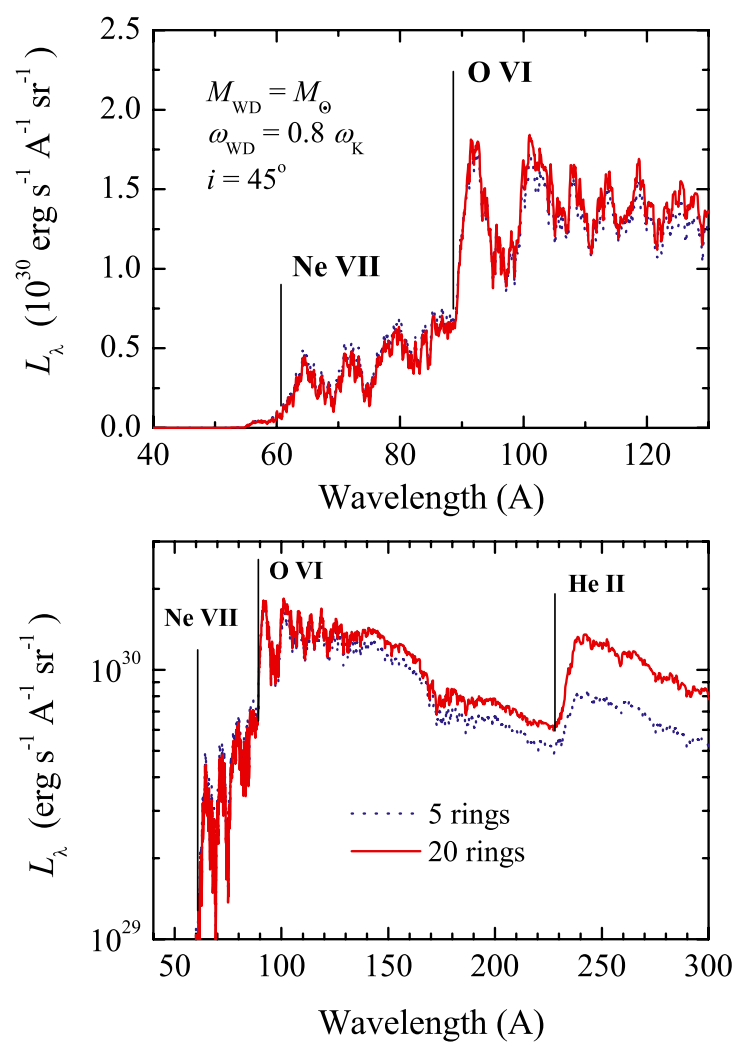

Fig. 6. Comparison of the spectra of the same BL model (see Fig. 4) computed using five (dotted curve) and twenty (solid curve) rings.

the fact that the atmosphere-model intensities compared to the blackbody spectra are different for low and high inclination angles. This is the result of different limb-darkening laws for the ring atmospheres (the intensity is mainly concentrated along the normal) compared to the isotropic blackbody radiation.

We investigated the importance of the number of rings to describe the model spectra. In addition to the spectrum of the $\mathrm{BL}$ reference model presented above, we computed the spectrum of the same model using five rings. A comparison of both spectra is shown in Fig. 6. The difference is significant at wavelengths longer than 120-130 $\AA$, but the spectra are almost identical at the shorter wavelengths. Our work was particularly motivated by the Chandra X-ray spectrum of SS Cyg in outburst (Mauche 2004). The grating spectra cover the wavelength range $10-130 \AA$, therefore, to model the observed BL spectra five rings are sufficient. Moreover, even the hottest ring spectrum alone can approximate the integral BL spectrum with a relatively good accuracy (see Fig. 7). All BL spectra of the two grids described above were computed using five rings.

It is important to explore the dependence of the BL model spectra on the BL parameters. For this purpose, we computed spectra of two model grids. In the first one we fixed the relative WD angular velocity $\omega_{\mathrm{WD}}=0.8 \omega_{\mathrm{K}}$ and investigated how the spectra depend on the WD mass $M_{\mathrm{WD}}$. In the second set we fixed the WD mass $M_{\mathrm{WD}}=M_{\odot}$ and varied the relative WD angular velocity. The obtained results are described below.

A comparison of the computed integral BL spectra for various parameters is shown in Figs. 8-10. The basic differences of the presented spectra are determined by differences in the BL model effective temperatures (see e.g. Fig. 8, top panel). The radial widths of all the computed models are similar (see the same figure and Hertfelder et al. 2013), but the luminosities can
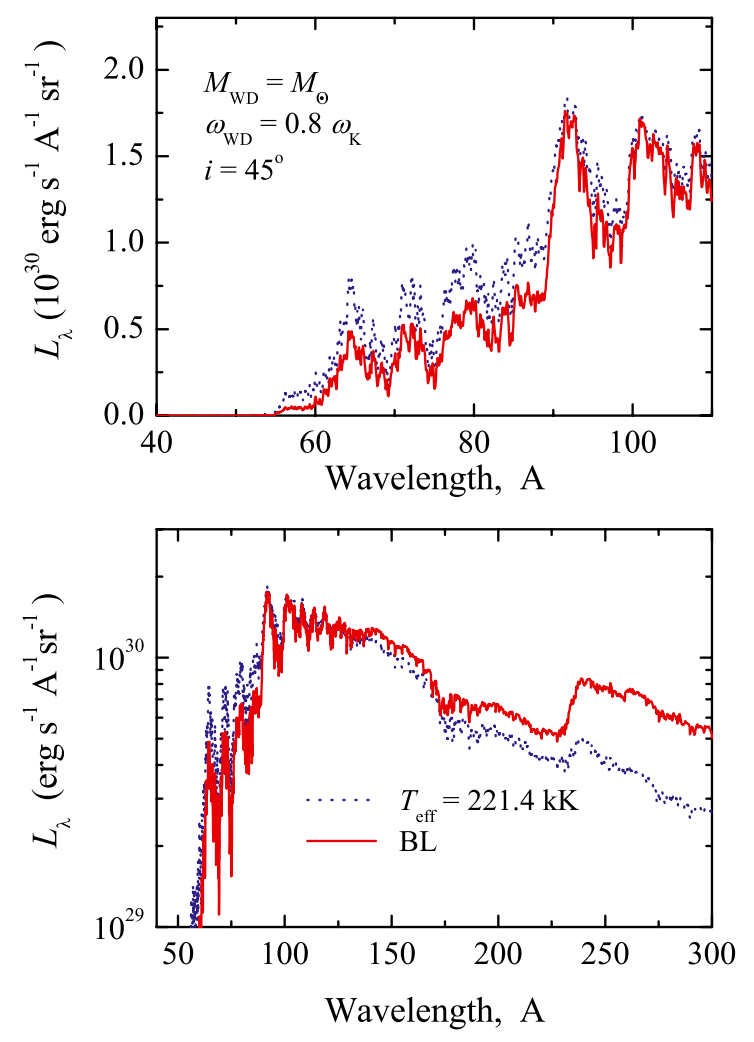

Fig. 7. Comparison of the BL model spectrum from Fig. 4 (solid curve) with the spectrum of the hottest ring (dotted curve) normalized to the flux at $100 \AA$.
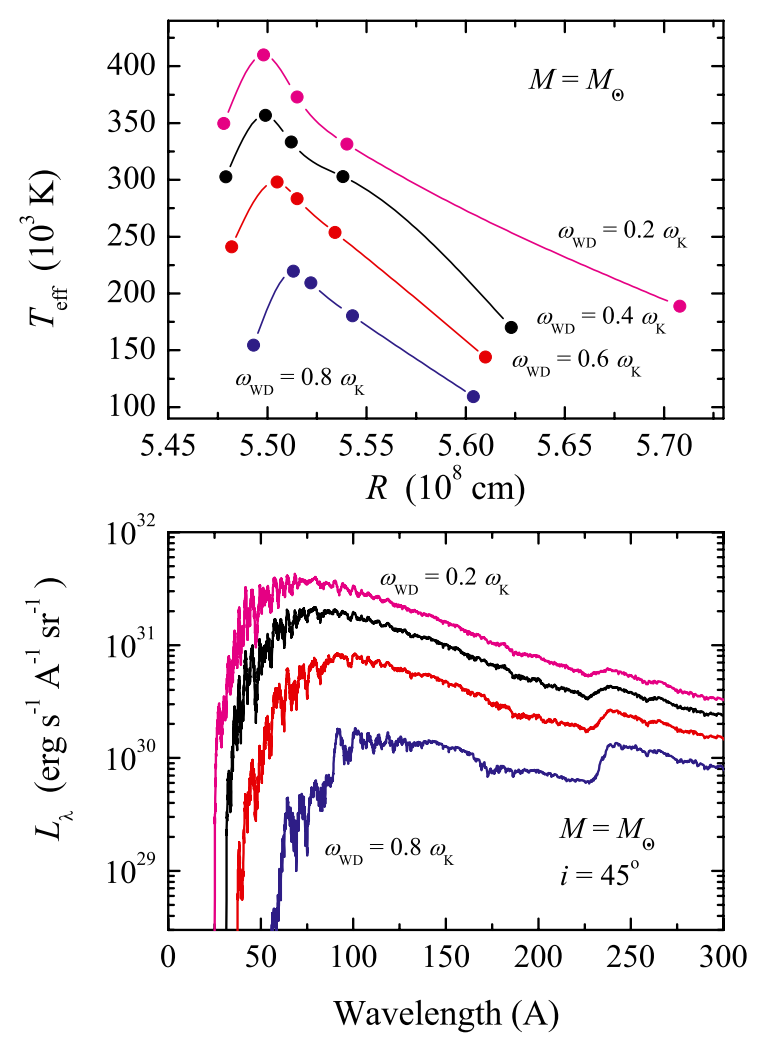

Fig. 8. Effective temperature distributions (top panel, the temperatures of the considered rings are marked by dots) and model spectra (bottom panel) for the models with different angular velocities $(0.2,0.4,0.6$ and $\left.0.8 \omega_{\mathrm{K}}\right)$ and fixed WD mass $\left(M_{\mathrm{WD}}=M_{\odot}\right)$. 


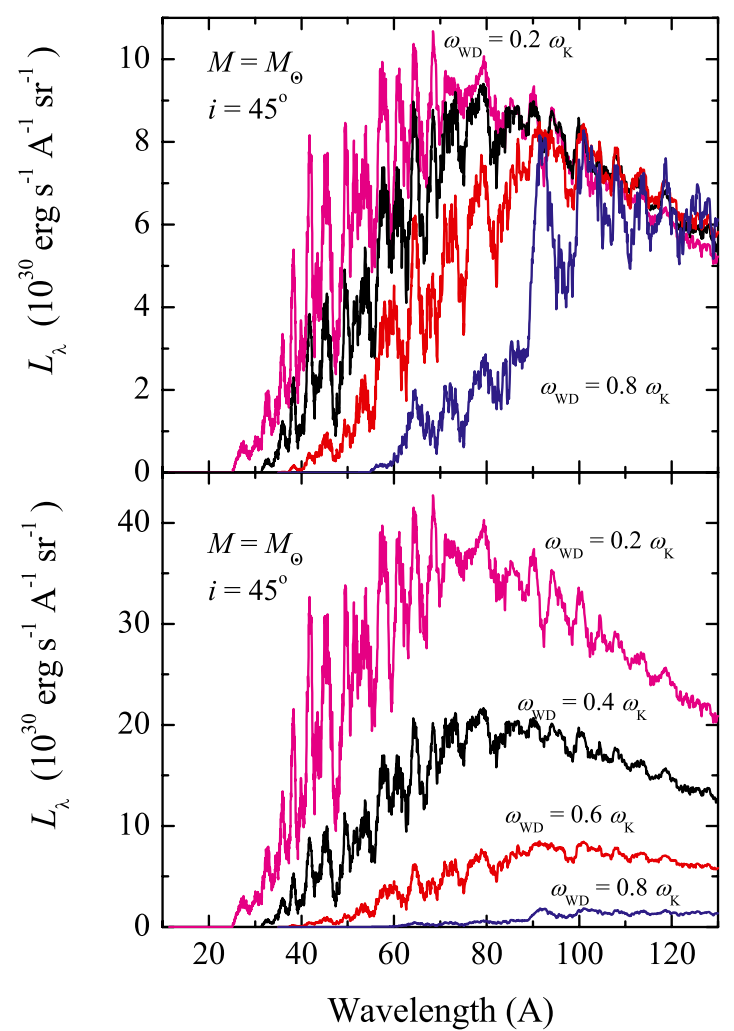

Fig. 9. Spectra of the same models as in Fig. 8 in the observed wavelength range. The spectra in the top panel are normalized to the $\omega_{\mathrm{WD}}=$ $0.6 \omega_{\mathrm{K}}$ model spectrum at $100 \AA$.
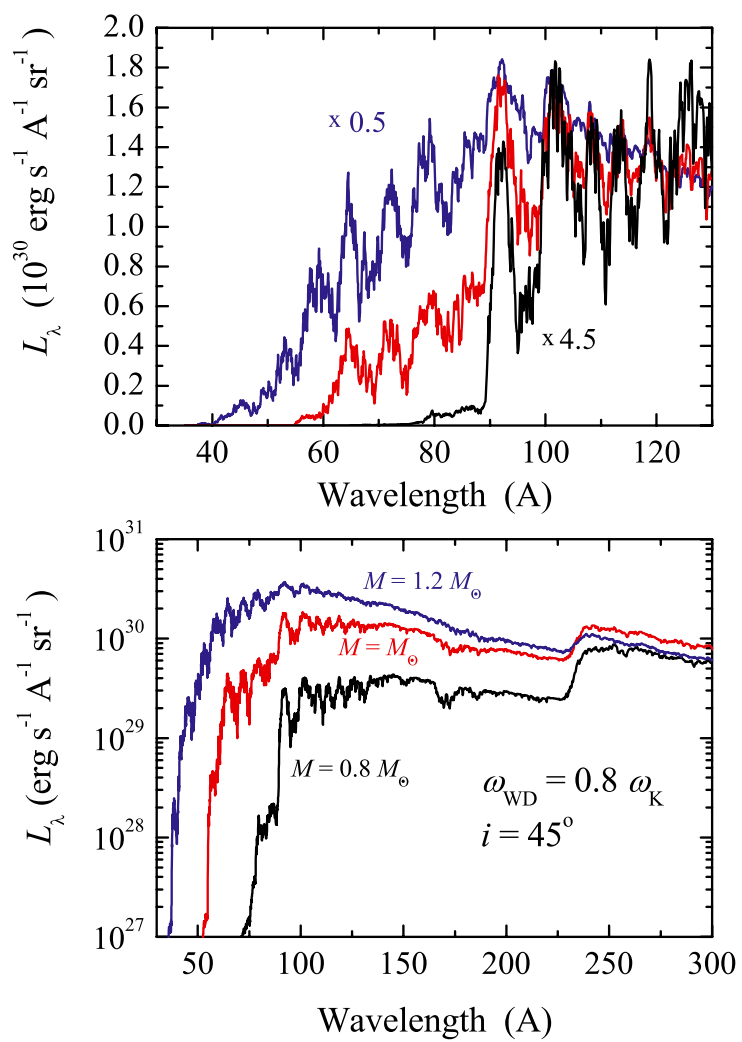

Fig. 10. Comparison of BL model spectra for three WD masses $(0.8,1$, $\left.1.2 M_{\odot}\right)$ and fixed relative angular velocity $\left(\omega_{\mathrm{WD}}=0.8 \omega_{\mathrm{K}}\right)$. The spectra in the top panel are normalized to the $M_{\mathrm{WD}}=M_{\odot}$ model flux at $100 \AA$. change more significantly. So, the BL effective temperatures directly connect with the BL luminosities, which are defined by mass accretion rate $\dot{M}$, WD mass, and relative WD angular velocity $\omega_{\mathrm{WD}} / \omega_{\mathrm{K}}$ (Kluźniak 1987; Kley 1991):

$L_{\mathrm{BL}}=\frac{1}{2} \frac{G M_{\mathrm{WD}} \dot{M}}{R_{\mathrm{WD}}}\left(1-\frac{\omega_{\mathrm{WD}}}{\omega_{\mathrm{K}}}\right)^{2}$.

All the models considered here have a fixed mass accretion rate. Therefore, luminosities and effective temperatures of these models only depend on WD masses and relative angular velocities. Both of them increase with the WD mass and decrease with the relative angular velocity. Higher BL effective temperatures have to correspond to harder spectra and higher ionization degree of the matter.

Our computations confirm these qualitative conclusions. Emergent spectra of the BL models around a solar mass WD with various relative angular velocities are shown in Fig. 8. The effective temperatures increase with decreasing WD relative angular velocity, and the emergent spectra become harder. As a result, the photo-absorption edges and the intensities of quasiabsorption and quasi-emission features are changing. In particular, the position of a notable spectral short-wavelength roll-off shifts from $60 \AA$ in the spectrum of BL model around the fastest rotating WD up to $25 \AA$ in the spectrum of the BL model around the slowest rotating WD. The same spectra in the 15-130 $\AA$ wavelength band on a linear flux scale are shown in Fig. 9. The spectra, which are normalized at $100 \AA$, are also shown in the top panel of the same Figure to emphasize the changes in the spectral shapes.

We note that some ring models (near the minimum of the surface density distribution) of the BL model around the nonrotating WD $\left(M_{\mathrm{WD}}=M_{\odot}, \omega_{\mathrm{WD}}=0\right)$ did not converge and a corresponding integral $\mathrm{BL}$ model spectrum was not computed. The reason is that these rings are effectively optically thin $\left(\tau_{\mathrm{eff}, v}=\right.$ $\left.\int_{0}^{\Sigma_{0} / 2} \sqrt{\sigma_{\mathrm{e}}\left(\sigma_{\mathrm{e}}+k_{v}\right)} \mathrm{d} m<1\right)$ over a wide wavelength range. We can see that the average Rosseland opacities for the hottest rings for the BL models around slowly rotating WDs are close to pure electron scattering and the input of the "true" opacity $k_{\mathrm{kr}}$ is negligible, because $\tau_{0} \approx \sigma_{\mathrm{e}} \Sigma_{0} / 2$ (see Table 1). Possibly, the similar effect may exist for other BL models around non-rotating WDs. It could be less important for the BL around WDs with lower masses and could be reduced for lesser mass accretion rates.

The difference between BL spectra computed for the fixed WD relative angular velocity but various WD masses are qualitatively similar (Fig. 10). The spectrum of the BL model around the heaviest WD is harder than the spectra of BL models around less heavy WDs.

\section{Comparison with observations}

As mentioned in the introduction, there are only four dwarf novae with observed soft X-ray/EUV radiation in outbursts, which can be associated with optically thick BLs. One of them is SS Cyg (Mauche 2004; Suleimanov et al. 2013). Fitting the spectrum gave effective temperature estimations of 190 (250) kK and a bolometric BL luminosity $18(5) \times 10^{33} \mathrm{erg} \mathrm{s}^{-1}$ for an assumed distance of $160 \mathrm{pc}$. The bolometric disk luminosity of SS Cyg at the peak of the outburst, $L_{\mathrm{D}} \approx 10^{35} \mathrm{erg} \mathrm{s}^{-1}$, was estimated by Mauche (2004) using the same distance and the data published by Polidan \& Holberg (1984). We note that the soft X-ray spectrum of SS Cyg was obtained by Chandra at the outburst peak, too. The corresponding mass accretion rate 
Table 4. Parameters of the strongest emission lines, preliminarily identified in the subtracted spectrum.

\begin{tabular}{llll}
\hline \hline$\lambda, \AA$ & Ion & Transition & $g f$ \\
\hline 49.12 & S IX & $2 \mathrm{p}^{4}{ }^{3} \mathrm{P}_{2}-3 \mathrm{~d}^{3} \mathrm{D}_{3}^{o}$ & 1.12 \\
65.7 & Al IX & $2 \mathrm{p}^{3}{ }^{2} \mathrm{P}_{3 / 2}-3 \mathrm{~d}^{2} \mathrm{D}_{5 / 2}$ & 1.33 \\
76.77 & Mg VIII & $2 \mathrm{p}^{3}{ }^{4} \mathrm{~S}^{\mathrm{o}}-3 \mathrm{~d}^{4} \mathrm{P}$ & $4.7^{a}$ \\
80.5 & Si VI & $2 \mathrm{p}^{5}{ }^{2} \mathrm{P}^{\mathrm{o}}-3 \mathrm{~d}^{2} \mathrm{P}$ & $2.3^{a}$ \\
83.97 & Mg VII & $2 \mathrm{p}^{2}{ }^{3} \mathrm{P}-3 \mathrm{~d}^{3} \mathrm{D}^{\mathrm{o}}$ & $\sim 7^{b}$ \\
85.41 & Mg VII & $2 \mathrm{p}^{2}{ }^{1} \mathrm{D}-3 \mathrm{~d}^{1} \mathrm{~F}^{\mathrm{o}}$ & 4.23 \\
88.1 & Ne VIII & $2 \mathrm{~s}^{2} \mathrm{~S}-3 \mathrm{p}^{2} \mathrm{P}^{\mathrm{o}}$ & $0.6^{a}$ \\
98.26 & Ne VIII & $2 \mathrm{p}^{2} \mathrm{P}_{3 / 2}^{\mathrm{o}}-3 \mathrm{~d}^{2} \mathrm{D}_{5 / 2}$ & 2.28 \\
106.14 & Ne VII & $2 \mathrm{p}^{3} \mathrm{P}^{\circ}-3 \mathrm{~d}^{3} \mathrm{D}$ & $4.3^{a}$ \\
107.94 & Al V & $2 \mathrm{p}^{5} \mathrm{P}_{3 / 2}^{\mathrm{o}}-3 \mathrm{~d}^{2} \mathrm{D}_{5 / 2}$ & 1.4 \\
\hline
\end{tabular}

Notes. Part of them could be blends with other lines. Data were taken from the CHIANTI database (Dere et al. 1997). ${ }^{(a)}$ Doublet, the $g f$ values are summed. ${ }^{(b)}$ Triplet, the $g f$ values are summed.

$\dot{M} \approx 1.5 \times 10^{-8} M_{\odot} / \mathrm{yr}$, which was used for the computation of all the BL models in this work, was obtained using this luminosity for the adopted WD parameters, $M_{\mathrm{WD}}=M_{\odot}$ and $R_{\mathrm{WD}}=5.5 \times 10^{8} \mathrm{~cm}$. The corresponding ratio of BL luminosity $L_{\mathrm{BL}}$ to disk luminosity $L_{\mathrm{D}}$ is $0.18(0.05)$. These values correspond to the fit of the spectrum by a hot stellar atmosphere that is close to Eddington limit (with $\log g=6.2$, Suleimanov et al. 2013), and the values in brackets were obtained using blackbody fits with broad absorption lines (Mauche 2004). The recent determination of SS Cyg's distance by Nelan \& Bond (2013) gave a lesser value, $\sim 110 \mathrm{pc}$ instead of $\sim 160 \mathrm{pc}$. It reduces the disk and the BL luminosities mentioned before, but does not change their ratio. The low value of $L_{\mathrm{BL}} / L_{\mathrm{D}}$ supports the hypothesis about a fast WD rotation in SS Cyg (Mauche 2004). In the ultraviolet spectrum of this CV in quiescence the absorption lines, which are in principle available to determine the WD's projected rotation velocity, were not found (Sion et al. 2010). The most probable reason for this finding are strong emission lines in the UV spectrum of SS Cyg in quiescence (Long et al. 2005), which could mask absorption lines in the WD spectrum. But it is necessary to keep in mind that the BL luminosity estimations are very model dependent and have large uncertainties.

We compare our BL model spectra to the observed Chandra spectrum of SS Cyg (Mauche 2004), using the well known normalization for converting the calculated spectra to the spectrum at Earth

$f_{\lambda}=\frac{L_{\lambda}}{d^{2}}$

where $d$ is the distance. A relatively good fit was obtained for the model spectrum of a BL with parameters $M_{\mathrm{WD}}=M_{\odot}, \omega_{\mathrm{WD}}=$ $0.8 \omega_{\mathrm{K}}$ for the distance $d \approx 107 \mathrm{pc}$ and the absorption by the neutral interstellar medium with $N_{\mathrm{H}} \approx 7 \times 10^{19} \mathrm{~cm}^{-2}$ (Fig. 11). We see that the model spectrum describes some prominent features in the observed spectrum, but there are also some additional features. The subtracted (observed minus BL model) spectrum (Fig. 11, bottom panel) is very similar to a spectrum of an optically thin hot plasma with a strong emission continuum of NeVII and numerous broad emission lines. Part of them are identified (see Fig. 11 and Table 4) as spectral lines with great $g f$ values. It is most probable that the difference spectrum is due to an optically thin outflow, which is projected on the cool WD and the accretion disc, see Fig. 12. We also show in the bottom of Fig. 11 the absorbed (with the same $N_{\mathrm{H}}$ ) spectrum of the homogeneous slab computed in LTE approximation with temperature

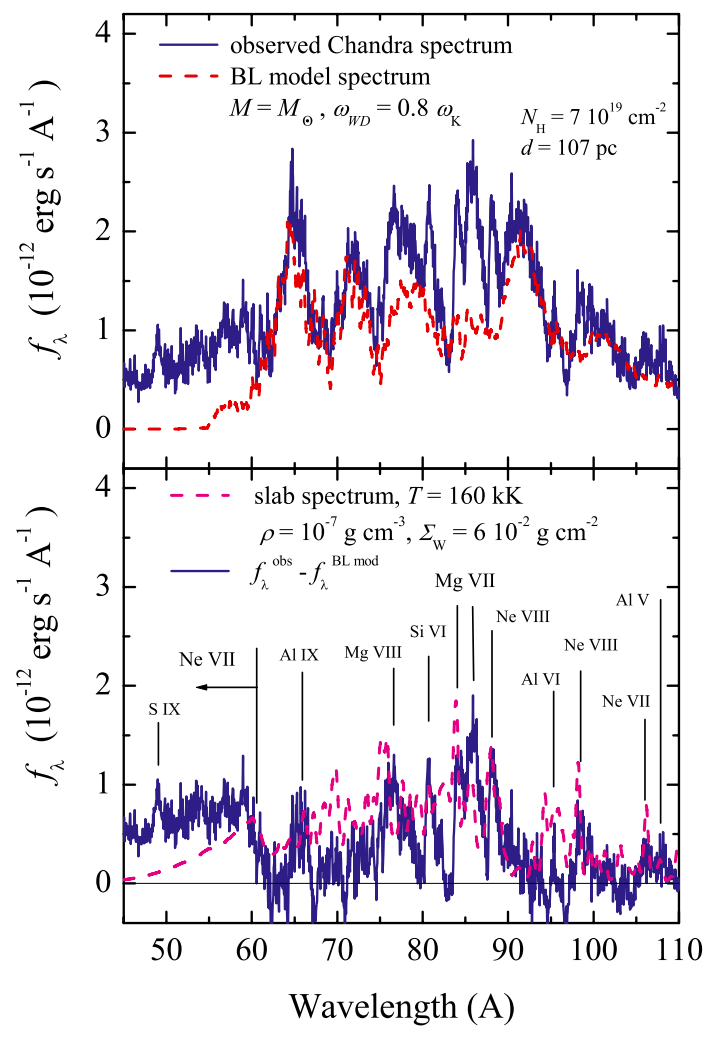

Fig. 11. Top panel: comparison of the observed SS Cyg soft X-ray spectrum (solid curve) with the BL model spectrum for a $1 M_{\odot} \mathrm{WD}$ and relative angular velocity $\omega_{\mathrm{WD}}=0.8 \omega_{\mathrm{K}}$ (dashed curve). Bottom panel: subtracted (observed minus BL model) spectrum (solid curve) together with the homogeneous slab model spectrum (dashed curve). The emission continuum of Ne VII below $60 \AA$ and the strongest identified emission lines are marked.

$T=160 \mathrm{kK}$, density $\rho=10^{-7} \mathrm{~g} \mathrm{~cm}^{-3}$, and surface density $\Sigma_{\mathrm{W}}=6 \times 10^{-2} \mathrm{~g} \mathrm{~cm}^{-2}$. The corresponding geometrical thickness of the slab is $6 \times 10^{5} \mathrm{~cm}$. The spectrum is computed by the short characteristics method and broadened using a Gauss function with $\sigma=0.2 \AA$. This model slab spectrum cannot fit all the emission details correctly, because the real outflow has to be strongly inhomogeneous, and, probably, the LTE approximation is not correct there. But it reproduces the general shape of the subtracted spectrum supporting our hypothesis. We found that the emission area of this slab is approximately four times greater than the visible model BL area. If we assume that the obtained density of the slab is correct for the averaged density of the outflow, we can estimate a total mass loss rate of $\dot{M}_{\mathrm{W}} \sim$ $\rho v_{\mathrm{esc}} S_{\mathrm{BL}} \sim 10^{-7} \mathrm{~g} \mathrm{~cm}^{-3} \times \sim 10^{8} \mathrm{~cm} \mathrm{~s}^{-1} \times \sim 10^{16}-10^{17} \mathrm{~cm}^{2}$ $\sim 10^{17}-10^{18} \mathrm{~g} \mathrm{~s}^{-1}$. This guess is very crude, but, nevertheless, it is comparable with the mass accretion rate, and, therefore, future BL models have to be computed with mass loss taken into account.

However, we have to point out that a recent estimation gave a lesser WD mass in SS Cyg, $M_{\mathrm{WD}}=0.81 \pm 0.18 M_{\odot}$ (Bitner et al. 2007) instead of $1 M_{\odot}$. We may expect that some BL model around a slower rotating WD with $M_{\mathrm{WD}}=0.8 M_{\odot}$ can have similar effective temperatures (and a similar spectrum) as the considered BL model with $M_{\mathrm{WD}}=M_{\odot}$ and $\omega_{\mathrm{WD}}=0.8 \omega_{\mathrm{K}}$. Indeed, we find that the spectra of BL models with parameters $M_{\mathrm{WD}}=0.8 M_{\odot}, \omega_{\mathrm{WD}}=0.6 \omega_{\mathrm{K}}$ and $M_{\mathrm{WD}}=M_{\odot}, \omega_{\mathrm{WD}}=0.8 \omega_{\mathrm{K}}$ are very similar (see Fig. 13). Therefore the spectrum of the BL model with parameters $M_{\mathrm{WD}}=0.8 M_{\odot}, \omega_{\mathrm{WD}} \approx 0.6 \omega_{\mathrm{K}}$ also has to fit the observed SS Cyg soft X-ray spectrum in the outburst 


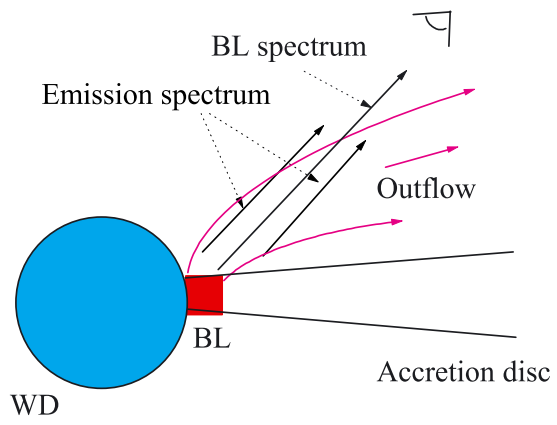

Fig. 12. Scheme of the BL outflow.

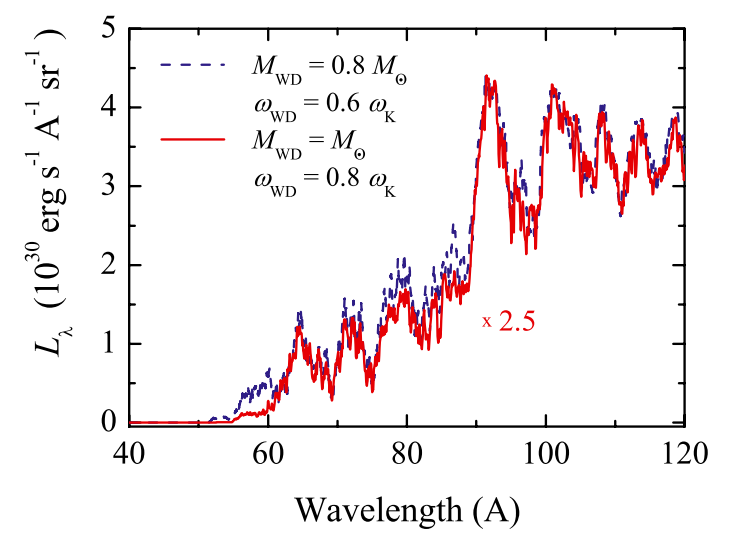

Fig. 13. Comparison of the BL model spectra for a $0.8 M_{\odot} \mathrm{WD}$ and relative angular velocity $\omega_{\mathrm{WD}}=0.6 \omega_{\mathrm{K}}$ (dashed curve) and for a $1 M_{\odot}$ $\mathrm{WD}$ and relative angular velocity $\omega_{\mathrm{WD}}=0.8 \omega_{\mathrm{K}}$ (solid curve). The flux of the latter spectrum is multiplied by 2.5 .

with $d \approx 170$ pc. The ratios $L_{\mathrm{BL}} / L_{\mathrm{D}}$ are 0.04 and 0.16 for these BL models, respectively, and they are close to the estimations obtained by Mauche (2004) and Suleimanov et al. (2013). Therefore, the BL model spectra computed here can fit the soft $\mathrm{X}$-ray spectrum of SS Cyg in outburst.

The second bright dwarf nova that exhibited a prominent soft X-ray/EUV flux during outburst is U Gem (Cordova et al. 1984; Long et al. 1996). But the properties of this emission differ significantly from the case of SS Cyg. A blackbody fit gives a temperature about $140 \mathrm{kK}$ and a BL luminosity of about $4 \times 10^{34} \mathrm{erg} \mathrm{s}^{-1}$ (for the distance $90 \mathrm{pc}$, Marsh et al. 1990) with the corresponding ratio $L_{\mathrm{BL}} / L_{\mathrm{D}} \sim 0.5$ (Long et al. 1996). The earlier X-ray observations performed by the Einstein observatory gave similar relations between the blackbody temperatures and the observed fluxes of these CVs' soft X-ray spectra (Cordova et al. 1980a,b, 1984).

The estimated rotation velocity of the WD in this CV is very low $\left(<100 \mathrm{~km} \mathrm{~s}^{-1}\right.$, Sion et al. 1994) and the derived relative BL luminosity is in accordance with the expected relative BL luminosity (see Eq. (41)). But all 1D BL models including ours predict higher effective temperatures for this luminous BL, at least $200-300 \mathrm{kK}$, in contradiction with the observed temperature. This means that the BL emitting area in U Gem is about 5-10 times greater than in SS Cyg, i.e. the BL width can be tens of percent of the WD radius instead of a few percent. Therefore, a new BL model is necessary to explain this fact.

A correct theory of optically thick BLs has to explain the observational properties of both dwarf novae. A possible reason could be the 2D nature of BLs around slowly rotating WDs. For example, the BL matter can spread over the WD surface increasing the radiating area (Kley 1991; Fisker \& Balsara 2005; Balsara et al. 2009). Another possible physical mechanism is deposition of a significant part of BL energy deep into the WD (Kippenhahn \& Thomas 1978; Godon et al. 1995), as it was also considered for T Tau type stars (Bertout \& Regev 1992; Regev $\&$ Bertout 1995), with subsequent radiation of this energy by a significant part of the WD surface above and below the equatorial plane. An additional possibility was suggested by Idan \& Shaviv (1996), who proposed that a significant part of the energy released in the BL might be carried away by some outflow (wind).

The EUV spectrum of U Gem in outburst is very similar to the soft X-ray spectrum of SS Cyg in outburst. It also shows numerous broad absorption- and emission-like features, but a few strong emission lines are also detected (Long et al. 1996). The latter probably arise in a strong BL wind and they are visible due to a higher inclination of U Gem's orbital plane relative to the line of sight $\left(i \approx 70^{\circ}\right.$, Zhang \& Robinson 1987) in comparison with the inclination angle of SS Cyg $\left(i \approx 50^{\circ}\right.$, Bitner et al. 2007). The even more inclined SU UMa-type dwarf nova OY Car $\left(i \approx 83^{\circ}\right.$, Littlefair et al. 2008) shows an EUV spectrum dominated by prominent emission lines in super-outburst as presented by Mauche \& Raymond (2000). These authors evaluated the wind mass-loss rate $\left(\leq 10^{-10} M_{\odot} \mathrm{yr}^{-1}\right)$ and argued that this value cannot be explained by a line-driven disk wind. It is possible that a line-driven BL wind as presented in our calculations can help to resolve this problem. We suggest that radiationdriven winds are much more powerful for BLs around slowly rotating WDs because of much higher effective temperatures at the same emitting area (see, e.g., Table 2). Therefore, such winds could be optically thick like the winds of Wolf-Rayet stars (see, e.g. Nugis \& Lamers 2002; Gräfener \& Hamann 2005), and the visible $\mathrm{BL}$ photospheres could have much greater emitting areas in comparison with the expected BL model sizes. This would lead to significant reduction of the averaged BL effective temperature while saving the bolometric luminosity. This hypothesis can explain the observed properties of the BL in U Gem. We suggest to call this kind of BLs "photospheric radius expanded" (PRE) BLs. A detailed BL wind investigation would be necessary to test these ideas.

\section{Conclusions}

In this paper we presented the first attempt to compute the soft X-ray/EUV spectra of optically thick BL models in CVs using the model stellar-atmosphere method. We used the 1D hydrodynamic BL models calculated by Hertfelder et al. (2013) for three WD masses $\left(0.8,1\right.$, and $\left.1.2 M_{\odot}\right)$ and various values of relative angular velocity $\left(0,0.2,0.4,0.6\right.$, and $\left.0.8 \omega_{\mathrm{K}}\right)$. Two other parameters, the mass-accretion rate $\dot{M}=1.5 \times 10^{-8} M_{\odot} \mathrm{yr}^{-1}$ and the viscosity parameter $\alpha=0.01$ were fixed. Every BL model is divided into a few rings, and every ring model along the $z$-direction is computed using the model-atmosphere method. The total BL model spectra are summed over the ring spectra with Doppler effect taken into account.

The effective temperatures of the considered BL model rings range from $100 \mathrm{kK}$ to $400 \mathrm{kK}$. At these conditions hydrogen and helium are almost fully ionized and highly-charged ions of heavy elements such as carbon, oxygen, neon, magnesium, and silicon determine the opacities and the shape of the model emergent spectra. The local ring spectra with numerous absorption lines of these chemical elements together with absorption edges are smeared by the fast ring rotation and the final model BL spectra show relatively broad absorption- and emission-like 
features, which cannot be identified with any individual absorption or emission lines. The absorption-like features arise at the spectral parts with high number densities of strong absorption lines and/or near blue sides of absorption edges. The emissionlike features arise at spectral parts with a low number densities of absorption lines and/or near red sides of absorption edges. An additional smoothing of the spectra due to a finite spectral resolution of X-ray instruments has to be also considered to form these prominent features. The predominance of spectral lines in the BL opacities leads to strong BL line-driven winds, which manifest themselves in EUV observations. We developed a simple method to treat approximately the influence of a linedriven wind on the BL model structure. This approach is not selfconsistent and it is not sufficiently correct for an investigation of the wind properties, but it offers the possibility to compute the model emergent spectra.

The observed soft X-ray and EUV spectra of the dwarf novae SS Cyg and U Gem in outburst are very similar to the computed model BL spectra and exhibit numerous emissionand absorption-like features (Mauche 2004; Long et al. 1996). Moreover, we reveal that the soft X-ray Chandra spectrum of SS Cyg in outburst can be fitted satisfactorily by the spectrum of our BL models with $M_{\mathrm{WD}}=M_{\odot}, \omega_{\mathrm{WD}}=0.8 \omega_{\mathrm{K}}$, and $M_{\mathrm{WD}}=0.8 M_{\odot}, \omega_{\mathrm{WD}}=0.6 \omega_{\mathrm{K}}$. with the interstellar absorption parameter $N_{\mathrm{H}} \approx 7 \times 10^{19} \mathrm{~cm}^{-2}$, and the distances $\approx 107$ and $170 \mathrm{pc}$ correspondingly. The ratio of the observed BL luminosity to the disk luminosity of SS Cyg in outburst is consistent with the model parameters. The subtracted spectrum is a spectrum of a hot optically thin plasma spectrum and could be associated with the outflow from the BL surface with the mass loss rate $\sim 10^{17}-10^{18} \mathrm{~g} \mathrm{~s}^{-1}$, which is comparable with the assumed mass accretion rate. Therefore, future BL models have to be computed with the mass loss taken into consideration.

On the other hand, the observed EUV flux properties of $\mathrm{U}$ Gem in outburst are contradicting our model predictions. We suggest that a powerful radiation-driven wind from U Gem's BL could form an extended photospheric BL surface reducing the averaged effective temperature but saving the bolometric luminosity. The properties of this "photospheric radius expanded" BL could be very similar to the observed U Gem BL properties. Additional extended $\mathrm{BL}$ radiation-driven wind investigations are necessary to proof this hypothesis.

Acknowledgements. We thank Chris Mauche, who put the Chandra X-ray spectrum of SS Cyg at our disposal and who helped us to fit it by our models, and the anonymous referee for numerous helpful remarks. V.S. thanks DFG for financial support (grant SFB/Transregio 7 "Gravitational Wave Astronomy") and Russian Foundation of Fundamental Research (grant 12-02-97006-r-povolzhe-a). M.H. received financial support from the German National Academic Foundation (Studienstiftung des deutschen Volkes).

\section{References}

Balbus, S. A., \& Hawley, J. F. 1991, ApJ, 376, 214

Balsara, D. S., Fisker, J. L., Godon, P., \& Sion, E. M. 2009, ApJ, 702, 1536

Baskill, D. S., Wheatley, P. J., \& Osborne, J. P. 2005, MNRAS, 357, 626

Belyaev, M. A., \& Rafikov, R. R. 2012, ApJ, 752, 115

Belyaev, M. A., Rafikov, R. R., \& Stone, J. M. 2012, ApJ, 760, 22

Belyaev, M. A., Rafikov, R. R., \& Stone, J. M. 2013, ApJ, 770, 67

Bertout, C., \& Regev, O. 1992, ApJ, 399, L163

Bitner, M. A., Robinson, E. L., \& Behr, B. B. 2007, ApJ, 662, 564

Castor, J. I., Abbott, D. C., \& Klein, R. I. 1975, ApJ, 195, 157

Chandrasekhar, S. 1960, Proc. National Academy of Science, 46, 253

Collins, T. J. B., Helfer, H. L., \& Van Horn, H. M. 1998, ApJ, 508, L159

Collins, T. J. B., Helfer, H. L., \& Van Horn, H. M. 2000a, ApJ, 534, 934

Collins, T. J. B., Helfer, H. L., \& Van Horn, H. M. 2000b, ApJ, 534, 944

Cordova, F. A., Chester, T. J., Tuohy, I. R., \& Garmire, G. P. 1980a, ApJ, 235, 163
Cordova, F. A., Nugent, J. J., Klein, S. R., \& Garmire, G. P. 1980b, MNRAS, 190,87

Cordova, F. A., Chester, T. J., Mason, K. O., Kahn, S. M., \& Garmire, G. P. 1984, ApJ, 278, 739

Dere, K. P., Landi, E., Mason, H. E., Monsignori Fossi, B. C., \& Young, P. R. 1997, A\&AS, 125, 149

Done, C., Osborne, J. P., \& Beardmore, A. P. 1995, MNRAS, 276, 483

Drew, J., \& Verbunt, F. 1985, MNRAS, 213, 191

Fisker, J. L., \& Balsara, D. S. 2005, ApJ, 635, L69

Frank, J., King, A., \& Raine, D. J. 2002, Accretion Power in Astrophysics: 3rd edn. (Cambridge: Cambridge University Press)

Gilfanov, M., Revnivtsev, M., \& Molkov, S. 2003, A\&A, 410, 217

Godon, P. 1995a, MNRAS, 277, 157

Godon, P. 1995b, MNRAS, 274, 61

Godon, P. 1997, ApJ, 483, 882

Godon, P., \& Sion, E. M. 2011, PASP, 123, 903

Godon, P., Regev, O., \& Shaviv, G. 1995, MNRAS, 275, 1093

Godon, P., Sion, E. M., Levay, K., et al. 2012, ApJS, 203, 29

Gräfener, G., \& Hamann, W.-R. 2005, A\&A, 432, 633

Hertfelder, M., Kley, W., Suleimanov, V., \& Werner, K. 2013, A\&A, 560, A56

Hubeny, I., Hummer, D., \& Lanz, T. 1994, A\&A, 282, 151

Hummer, D., \& Mihalas, D. 1988, ApJ, 331, 794

Ibragimov, A. A., Suleimanov, V. F., Vikhlinin, A., \& Sakhibullin, N. A. 2003, Astron. Rep., 47, 186

Idan, I., \& Shaviv, G. 1996, MNRAS, 281, 604

Inogamov, N. A., \& Sunyaev, R. A. 1999, Astron. Lett., 25, 269

Inogamov, N. A., \& Sunyaev, R. A. 2010, Astron. Lett., 36, 848

Jones, M. H., \& Watson, M. G. 1992, MNRAS, 257, 633

Kato, S., \& Inagaki, S. 1994, PASJ, 46, 289

Kippenhahn, R., \& Thomas, H.-C. 1978, A\&A, 63, 265

Kley, W. 1989, A\&A, 222, 141

Kley, W. 1991, A\&A, 247, 95

Kley, W., \& Hensler, G. 1987, A\&A, 172, 124

Kluźniak, W. 1987, PhD thesis, Stanford University

Kriz, S., \& Hubeny, I. 1986, Bull. Astr. Inst. Czechosl., 37, 129

Kudritzki, R.-P., \& Puls, J. 2000, ARA\&A, 38, 613

Kurucz, R. L. 1970, SAO Special Report, 309

Lanz, T., Telis, G. A., Audard, M., et al. 2005, ApJ, 619, 517

Lewin, W. H. G., van Paradijs, J., \& Taam, R. E. 1993, Space Sci. Rev., 62, 223

Littlefair, S. P., Dhillon, V. S., Marsh, T. R., et al. 2008, MNRAS, 388, 1582

Long, K. S., Mauche, C. W., Raymond, J. C., Szkody, P., \& Mattei, J. A. 1996 ApJ, 469, 841

Long, K. S., Froning, C. S., Knigge, C., et al. 2005, ApJ, 630, 511

Long, K. S., Brammer, G., \& Froning, C. S. 2006, ApJ, 648, 541

Lynden-Bell, D., \& Pringle, J. E. 1974, MNRAS, 168, 603

Marsh, T. R., Horne, K., Schlegel, E. M., Honeycutt, R. K., \& Kaitchuck, R. H. 1990, ApJ, 364, 637

Mauche, C. W. 1996, in Cataclysmic Variables and Related Objects, eds. A. Evans \& J. H. Wood, Astrophys. IAU Colloq., 158, Space Sci. Library, 208, 243

Mauche, C. W. 2004, ApJ, 610, 422

Mauche, C. W., \& Raymond, J. C. 2000, ApJ, 541, 924

Mauche, C. W., Raymond, J. C., \& Mattei, J. A. 1995, ApJ, 446, 842

Mihalas, D. 1978, Stellar atmospheres (San Francisco: W. H. Freeman and Co.)

Mitsuda, K., Inoue, H., Koyama, K., et al. 1984, PASJ, 36, 741

Mukai, K., Kinkhabwala, A., Peterson, J. R., Kahn, S. M., \& Paerels, F. 2003, ApJ, 586, L77

Nagel, T., Dreizler, S., Rauch, T., \& Werner, K. 2004, A\&A, 428, 109

Narayan, R., Loeb, A., \& Kumar, P. 1994, ApJ, 431, 359

Nauenberg, M. 1972, ApJ, 175, 417

Nelan, E. P., \& Bond, H. E. 2013, ApJ, 773, L26

Nugis, T., \& Lamers, H. J. G. L. M. 2002, A\&A, 389, 162

Olson, G. L., \& Kunasz, P. B. 1987, J. Quant. Spectr. Rad. Transf., 38, 325

Patterson, J. 1981, ApJS, 45, 517

Patterson, J., \& Raymond, J. C. 1985a, ApJ, 292, 550

Patterson, J., \& Raymond, J. C. 1985b, ApJ, 292, 535

Piro, A. L., \& Bildsten, L. 2004a, ApJ, 616, L155

Piro, A. L., \& Bildsten, L. 2004b, ApJ, 610, 977

Polidan, R. S., \& Holberg, J. B. 1984, Nature, 309, 528

Popham, R. 1997, ApJ, 478, 734

Popham, R. 1999, MNRAS, 308, 979

Popham, R., \& Narayan, R. 1995, ApJ, 442, 337

Pringle, J. E. 1977, MNRAS, 178, 195

Pringle, J. E., \& Savonije, G. J. 1979, MNRAS, 187, 777

Prinja, R. K., \& Rosen, R. 1995, MNRAS, 273, 461

Proga, D., Stone, J. M., \& Drew, J. E. 1998, MNRAS, 295, 595

Rauch, T., Orio, M., Gonzales-Riestra, R., et al. 2010, ApJ, 717, 363 
A\&A 571, A55 (2014)

Regev, O. 1983, A\&A, 126, 146

Regev, O., \& Bertout, C. 1995, MNRAS, 272, 71

Revnivtsev, M. G., \& Gilfanov, M. R. 2006, A\&A, 453, 253

Revnivtsev, M. G., Burenin, R. A., Tkachenko, A. Y., et al. 2012, Astron. Lett., 38,238

Revnivtsev, M. G., Suleimanov, V. F., \& Poutanen, J. 2013, MNRAS, 434, 2355

Robertson, J. A., \& Frank, J. 1986, MNRAS, 221, 279

Robinson, E. L., \& Nather, R. E. 1979, ApJS, 39, 461

Shakura, N. I., \& Sunyaev, R. A. 1973, A\&A, 24, 337

Shaviv, G., \& Wehrse, R. 1991, A\&A, 251, 117

Sion, E. M., Long, K. S., Szkody, P., \& Huang, M. 1994, ApJ, 430, L53

Sion, E. M., Godon, P., Myzcka, J., \& Blair, W. P. 2010, ApJ, 716, L157

Suleimanov, V., \& Poutanen, J. 2006, MNRAS, 369, 2036

Suleimanov, V., \& Werner, K. 2007, A\&A, 466, 661

Suleimanov, V. F., Lipunova, G. V., \& Shakura, N. I. 2007, Astron. Rep., 51, 549

Suleimanov, V. F., Mauche, C. W., Zhuchkov, R. Y., \& Werner, K. 2013, in 18th European White Dwarf Workshop., eds. J. Krzesiński, G. Stachowski,

P. Moskalik, \& K. Bajan, ASP Conf. Ser., 469, 349

Suleymanov, V. F. 1992, Sov. Astron. Lett., 18, 104
Swank, J. H. 1979, in White Dwarfs and Variable Degenerate Stars, eds. H. M. van Horn, \& V. Weidemann, IAU Colloq., 53, 135

Tylenda, R. 1977, Acta Astron., 27, 235

Tylenda, R. 1981, Acta Astron., 31, 267

van der Klis, M. 1989, ARA\&A, 27, 517

van der Klis, M. 2000, ARA\&A, 38, 717

van Teeseling, A., \& Verbunt, F. 1994, A\&A, 292, 519

van Teeseling, A., Beuermann, K., \& Verbunt, F. 1996, A\&A, 315, 467

Velikhov, E. 1959, Sov. Phys. -JETP, 36, 1398

Verner, D. A., \& Yakovlev, D. G. 1995, A\&AS, 109, 125

Verner, D. A., Ferland, G. J., Korista, K. T., \& Yakovlev, D. G. 1996, ApJ, 465, 487

Wade, R. A., \& Hubeny, I. 1998, ApJ, 509, 350

Warner, B. 1986, Ap\&SS, 118, 271

Warner, B. 2003, Cataclysmic Variable Stars (Cambridge: Cambridge University Press)

Warner, B., \& Pretorius, M. L. 2008, MNRAS, 383, 1469

Warner, B., \& Woudt, P. A. 2002, MNRAS, 335, 84

Zhang, E.-H., \& Robinson, E. L. 1987, ApJ, 321, 813 